MATHEMATICS OF COMPUTATION

Volume 78, Number 266, April 2009, Pages 739-770

S 0025-5718(08)02181-9

Article electronically published on November 6, 2008

\title{
ON ESTIMATORS FOR EIGENVALUE/EIGENVECTOR APPROXIMATIONS
}

\author{
LUKA GRUBIŠIĆ AND JEFFREY S. OVALL
}

\begin{abstract}
We consider a large class of residuum based a posteriori eigenvalue/eigenvector estimates and present an abstract framework for proving their asymptotic exactness. Equivalence of the estimator and the error is also established. To demonstrate the strength of our abstract approach we present a detailed study of hierarchical error estimators for Laplace eigenvalue problems in planar polygonal regions. To this end we develop new error analysis for the Galerkin approximation which avoids the use of the strengthened CauchySchwarz inequality and the saturation assumption, and gives reasonable and explicitly computable upper bounds on the discretization error. A brief discussion is also given concerning the design of estimators which are in the same spirit, but are based on different a posteriori techniques-notably, those of gradient recovery type.
\end{abstract}

\section{INTRODUCTION}

The purpose of this paper is to analyze a posteriori eigenvalue/eigenvector estimators for a class of positive definite symmetric eigenvalue problems. We reduce the study of the eigenvalue/eigenvector estimators to the study of associated boundary value problems and reuse available results on the a posteriori error analysis for those auxiliary problems. In particular, we consider those estimators for boundary value problems which are asymptotically exact (under certain conditions, cf. [5, 6, 30]) and show that under a natural convergence and nondegeneracy assumption on the spectral approximation problems our derived eigenvalue/eigenvector estimators are also asymptotically exact.

Our analysis also yields equivalence of the a posteriori estimator and the relative eigenvalue/eigenvector error with reasonable and computable equivalence constants. Our results are based on the techniques of the relative perturbation theory from Numerical Linear Algebra - we are particularly influenced by the approach of [13 - which have recently been considered in [16, 18, 19] in the setting of infinite dimensional Hilbert spaces.

Estimators for the adaptive finite element eigenvalue approximations have recently been considered in literature from several viewpoints. One possible approach is that of Heuveline and Rannacher [22] and Verfürth 34] which is based on a general analysis of the nonlinear (single vector) residuum equations. Such approaches make an analysis of the approximations of multiple eigenvalues somewhat more

Received by the editor February 20, 2007 and, in revised form, April 18, 2008.

2000 Mathematics Subject Classification. Primary 65N25; Secondary 65N50, 65N15, 65N30.

Key words and phrases. Eigenvalue problem, finite element method, a posteriori error estimates.

(C)2008 American Mathematical Society Reverts to public domain 28 years from publication 
involved. On the other hand, the approaches of Neymeyr 29, Durán, Padra and Rodríguez [14, Larson [24] and Mao, Shen and Zhou [26] analyze the same residual equations directly. Eigenvalue estimates for multiple eigenvalues and the associated invariant subspaces are then derived by maximizing the residual estimate over the approximate test subspace. The approach of 14 is essentially asymptotic in nature since the equivalence is shown up to the higher order terms. The analysis of when these higher order terms may be neglected is given, but the equivalence results are still not constructive in nature. The analysis of 24 is performed by a combination of a posteriori and a priori analysis and it unfortunately requires that the associated boundary value problem be $H^{2}$ regular.

On the other hand, we start from the abstract block matrix residual equation for the invariant subspace - as presented in [19] - which allows a natural treatment of the eigenvalue multiplicity without incurring unnecessary regularity constraints. This error representation formula is used both to prove the equivalence (with explicit and reasonable constants) of the residuum based estimator as well as its asymptotic exactness. We also indicate that there is a class of $\sin \Theta$-type theorems which use the same type of residual measures to obtain computable bounds on the invariant subspace error; see 20. In this paper we do not quote those results explicitly (an application of the results from 20 in our context is straightforward), but rather concentrate on obtaining estimates of the norm of the gradient of the eigenvector error. Of all the approaches which we have mentioned the closest in spirit to our considerations are those from 29 and 26 since they both reduce the study of the eigenvalue problem on the study of the associated boundary value problem.

More to the point, we use a similar preconditioned hierarchical error estimator to the one which is used in 29] and prove that our modified estimator is not only rigorous/reliable but also efficient; in other words, equivalent to the error. Eigenvector error estimates, which were not considered in [29] are also given. Furthermore, we provide reasonable and computable equivalence constants for both eigenvalue and eigenvector error. The authors of [26] analyze local averaging type error estimators and prove their asymptotic exactness. To illustrate the generality of our block-matrix (invariant subspace) residual equations we briefly discuss how to obtain similar results for some other gradient recovery type error estimators.

In our detailed analysis of the Dirichlet Laplace eigenvalue problem we wanted to reuse the known results on the error of the Galerkin approximation. However, the available estimates did not suit our needs, since in the eigenvalue problem we wanted to simultaneously consider the associated boundary value problem for a large class of right-hand side vectors. The standard estimates involved constants which were intricately dependent on the right-hand side vector and it was not possible to decouple this dependence easily. Therefore, we have developed a new error analysis - which is interesting in its own right - for the Galerkin approximation which avoids the use of the strengthened Cauchy-Schwarz inequality and the saturation assumption. Furthermore, this analysis yields reasonable and explicitly computable upper bounds on the discretization error.

The theory of [16, 18, 19] has been developed - in the framework of the perturbation theory from [23, Chapters VI-VIII] - for an abstract positive definite symmetric and closed form $h$ in a general Hilbert space $\mathcal{H}$. We use this abstract approach 
to establish the asymptotic exactness of the scaled residual and the relative eigenvalue/eigenvector error in Section 4. It is often the case that one loses information about important specific examples by making general abstract arguments. However, to show the strength of our theory we focus on the Dirichlet Laplacian in polygonal domains with possibly reentrant corners, demonstrating that nothing important is lost in the general arguments. In Section 3 we introduce our measures of the size of the scaled residual - which we call approximation defects - and give a detailed constructive (equivalence) analysis of their behaviour. Furthermore, to this end in Section 5 we revisit the class of error estimators for boundary value problems from [12, 27, 28, and obtain new reasonable and computable upper bounds on the discretization error.

\section{NotATION AND PRELIMINARIES}

Let $\mathcal{R} \subset \mathbb{R}^{2}$ be a bounded polygonal region, possibly with reentrant corners. By $H_{0}^{1}(\mathcal{R})$ we denote the subspace of the first order Sobolev space $H^{1}(\mathcal{R})$ which consists of all those functions which vanish on the boundary $\partial \mathcal{R}$ (this is meant in the sense of the trace operator $)$. The space $H_{0}^{1}(\mathcal{R})$ is assumed to be equipped with the norm $\|u\|_{H_{0}^{1}}=|u|_{1,2}$. By $\|\cdot\|$ we always denote the norm on $L^{2}(\mathcal{R})$ and we use $|\cdot|_{k, 2}, k \in \mathbb{N}$ to denote the standard Sobolev semi-norms. For a subdomain $\mathcal{S} \subset \mathcal{R}$ we use $\|\cdot\|_{\mathcal{S}}$ to denote the $L^{2}$ norm on $\mathcal{S}$. For other real $\alpha \in(0,1]$ we also use $H^{1+\alpha}(\mathcal{R})$ to denote standard interpolation spaces.

In Section 4 we shall deal with variational eigenvalue problems for a general closed symmetric and positive definite form $h$ in a Hilbert space in the sense of 23. Theorem VI-2.23, p. 331]. Indeed, this is a natural framework for most of our theory and this is the generality in which the results of [19] have been proved. However, in this paper it is our aim to discuss the finer properties of the construction from [19. To this end, and also to ease the presentation, we concentrate on the Dirichlet Laplace eigenvalue problem. In the weak form this reads: Find the real eigenvalue $\lambda$ and a nonzero eigenfunction $v \in H_{0}^{1}(\mathcal{R})$ such that

$$
\left\{\begin{aligned}
\int_{\mathcal{R}} \nabla v \cdot \nabla \psi & =\lambda \int_{\mathcal{R}} v \psi, \quad \text { for all } \psi \in H_{0}^{1}(\mathcal{R}), \\
\int_{\mathcal{R}}|v|^{2} & =1 .
\end{aligned}\right.
$$

Problem (2.1) is attained by a sequence of positive eigenvalues $\lambda_{i}$-ordered in the ascending order $\lambda_{i} \leq \lambda_{i+1}, i \in \mathbb{N}$ according to multiplicity-such that $\lambda_{i} \rightarrow \infty$ and a sequence of associated eigenvectors such that $v_{j} \in H^{1+\alpha}(\mathcal{R})$ (the parameter $\alpha$ depends on the regularity of $\mathcal{R}$ ). In the operator form this can be written as

$$
\left\{\begin{aligned}
-\triangle v_{i} & =\lambda_{i} v_{i}, & & \text { in } \mathcal{R}, \\
v_{i} & =0 & & \text { on } \partial \mathcal{R} .
\end{aligned}\right.
$$

The gradient operator $\nabla$ and the Laplace operator $\triangle$ are meant in the distributional sense. We will also use the notation $-\triangle$ to denote the positive definite self-adjoint operator $\mathbf{H}$ which represents the symmetric form

$$
h(\psi, \phi)=\int_{\mathcal{R}} \nabla \psi \cdot \nabla \phi, \quad \psi, \phi \in H_{0}^{1}(\mathcal{R})
$$

in the sense of [23, Theorem VI-2.23, p. 331], i.e. $h(\psi, \phi)=\left(\mathbf{H}^{1 / 2} \psi, \mathbf{H}^{1 / 2} \phi\right)$, $\psi, \phi \in H_{0}^{1}(\mathcal{R})$ and the domain of definition of $\mathbf{H}^{1 / 2}$ equals $H_{0}^{1}(\mathcal{R})$. The associated quadratic form is denoted by $h[\psi]=h(\psi, \psi)^{1 / 2}$. In this paper we use $\mathcal{D}(\mathbf{H})$ to 
denote the domain of the operator $\mathbf{H}, \Sigma(\mathbf{H})$ to denote its spectrum and $\mathcal{Q}(h)$ to denote the domain of the symmetric form $h$. Furthermore, for a linear operator $A$ we use $\mathrm{R}(A)$ and $\mathrm{N}(A)$ to denote its range an the null space.

To compute finite element approximations for the eigenvalues and eigenvectors of (2.1) we define a family of finite element spaces. Let $\mathcal{T}_{d}$ be a collection of closed triangles such that $\overline{\mathcal{R}}=\bigcup_{\tau \in \mathcal{T}_{d}} \tau$. The diameter of a triangle $\tau \in \mathcal{T}_{d}$ is given by $d_{\tau}$, and the maximal diameter

$$
d=\max _{\tau \in \mathcal{T}_{d}} d_{\tau}
$$

is used to index the triangulation. We will only consider conforming triangulations $\mathcal{T}_{d}$ of $\mathcal{R}$ - triangulations such that the intersection of any two triangles in $\mathcal{T}_{d}$ is either empty, or consists of a common edge or vertex. For a given triangulation $\mathcal{T}_{d}$ we define the finite dimensional function spaces:

$$
\begin{aligned}
& \mathfrak{L}\left(\mathcal{T}_{d}\right)=\left\{u \in H_{0}^{1}(\mathcal{R}) \mid \text { for } T \in \mathcal{T}_{d},\left.u\right|_{T} \text { is a linear function }\right\}, \\
& \mathfrak{Q}\left(\mathcal{T}_{d}\right)=\left\{u \in H_{0}^{1}(\mathcal{R}) \mid \text { for } T \in \mathcal{T}_{d},\left.u\right|_{T} \text { is a quadratic function }\right\} .
\end{aligned}
$$

We will also make use of the space $\mathfrak{B}\left(\mathcal{T}_{d}\right)$ of edge bubble functions, which are those functions from $\mathfrak{Q}\left(\mathcal{T}_{d}\right)$ which vanish at the vertices of all triangles in $\mathcal{T}_{d}$. We have the hierarchical decomposition $\mathfrak{Q}\left(\mathcal{T}_{d}\right)=\mathfrak{L}\left(\mathcal{T}_{d}\right) \oplus \mathfrak{B}\left(\mathcal{T}_{d}\right)$, so we use $\mathfrak{B}\left(\mathcal{T}_{d}\right)=$ $\mathfrak{Q}\left(\mathcal{T}_{d}\right) \ominus \mathfrak{L}\left(\mathcal{T}_{d}\right)$ as a compact definition.

We take the standard bases for $\mathfrak{L}\left(\mathcal{T}_{d}\right)$ and $\mathfrak{B}\left(\mathcal{T}_{d}\right)$, which are described as follows. Let $\mathcal{V}_{d}$ be the set of interior vertices, $\overline{\mathcal{V}}_{d}$ the set of all vertices, and $\mathcal{E}_{d}$ the set of the interior edges in the triangulation $\mathcal{T}_{d}$. Then the bases for $\mathfrak{L}\left(\mathcal{T}_{d}\right)$ and $\mathfrak{B}\left(\mathcal{T}_{d}\right)$ are, respectively,

$$
\left\{\ell_{z} \in \mathfrak{L}\left(\mathcal{T}_{d}\right) \mid \ell_{z}\left(z^{\prime}\right)=\delta_{z z^{\prime}} \text { for } z \in \mathcal{V}_{d}, z^{\prime} \in \overline{\mathcal{V}}_{d}\right\}
$$

and

$$
\left\{b_{e} \in \mathfrak{B}\left(\mathcal{T}_{d}\right) \mid b_{e}=4 \ell_{z} \ell_{z^{\prime}} \text { for } e \in \mathcal{E}_{d} \text { with endpoints } z, z^{\prime}\right\} .
$$

The factor of 4 in the definition of $b_{e}$ is chosen so that the coefficients of a function in $\mathfrak{B}\left(\mathcal{T}_{d}\right)$ with respect to this basis coincide with the values of the function at the midpoints of the corresponding edges. The union of these sets forms a (hierarchical) basis for $\mathfrak{Q}\left(\mathcal{T}_{d}\right)$. The cardinalities of the sets $\mathcal{T}_{d}, \mathcal{V}_{d}$ and $\mathcal{E}_{d}$ are related by Euler's formula, $\left|\mathcal{E}_{d}\right|=\left|\mathcal{T}_{d}\right|+\left|\mathcal{V}_{d}\right|-1$, and we generally expect that $\mathcal{E}_{d}$ has between three and four times the cardinality of $\mathcal{V}_{d}$. We will use the spaces $\mathfrak{L}\left(\mathcal{T}_{d}\right)$ to compute eigenvalue/eigenvector approximations and the spaces $\mathfrak{B}\left(\mathcal{T}_{d}\right)$ to assess the quality of the approximation.

A discrete variant of (2.1) now reads: Find nonzero $u \in \mathfrak{L}\left(\mathcal{T}_{d}\right)$ such that

$$
\left\{\begin{aligned}
\int_{\mathcal{R}} \nabla u \cdot \nabla \psi & =\lambda \int_{\mathcal{R}} u \psi, \quad \text { for all } \psi \in \mathfrak{L}\left(\mathcal{T}_{d}\right) \\
\int_{\mathcal{R}}|u|^{2} & =1,
\end{aligned}\right.
$$

and it is attained by a finite number of discrete eigenvalues $\lambda_{i}\left(\mathcal{T}_{d}\right)$ and discrete eigenvectors $u_{i}\left(\mathcal{T}_{d}\right), i=1, \ldots, \operatorname{dim} \mathfrak{L}\left(\mathcal{T}_{d}\right)$. The discrete eigenvalues $\lambda_{i}\left(\mathcal{T}_{d}\right)$ (discrete eigenvectors $u_{i}\left(\mathcal{T}_{d}\right)$ ) are often called the Ritz values/vectors. We reserve these terms for those discrete eigenvalues/eigenvectors which approximate a particular eigenvalue of interest and have a joint multiplicity which is equivalent to the multiplicity of the eigenvalue. 
Let us assume that we want to approximate the eigenvalue $\lambda_{q}$ of multiplicity $m \in \mathbb{N}$ of (2.1). This is to say we assume that

$$
\lambda_{q-1}<\lambda_{q}=\lambda_{q+1}=\cdots=\lambda_{q+m-1}<\lambda_{q+m} .
$$

We also assume that $q+m<\operatorname{dim} \mathfrak{L}\left(\mathcal{T}_{d}\right)$. By $P_{d}$ we denote the orthogonal projection onto the linear span of $\left\{u_{q}\left(\mathcal{T}_{d}\right), \ldots, u_{q+m-1}\left(\mathcal{T}_{d}\right)\right\}$. We use $\mathrm{R}\left(P_{d}\right)$ to denote the range of the projection $P_{d}$ and we write

$$
\mathrm{R}\left(P_{d}\right)=\operatorname{span}\left\{u_{q}\left(\mathcal{T}_{d}\right), \ldots, u_{q+m-1}\left(\mathcal{T}_{d}\right)\right\} .
$$

Given such a subspace $\mathrm{R}\left(P_{d}\right)$ we set $\mu_{i}^{d}=\lambda_{q-1+i}\left(\mathcal{T}_{d}\right)$ and $\psi_{i}^{d}=u_{q-1+i}\left(\mathcal{T}_{d}\right)$. We call $\mu_{i}^{d}$ and $\psi_{i}^{d}$ Ritz values/vectors from the subspace $\mathrm{R}\left(P_{d}\right)$. For $\mathrm{M} \in \mathbb{N}$ we also define the orthogonal projection $L_{\mathrm{M}}(d)$ as the orthogonal projection onto $\operatorname{span}\left\{u_{1}\left(\mathcal{T}_{d}\right), \ldots, u_{\mathrm{M}}\left(\mathcal{T}_{d}\right)\right\}$. Let us also note that in what follows when choosing our notation we will suppress the dependence on the parameter $d$ wherever there is no danger of confusion.

In the case when we have a general orthogonal projection $P, \mathrm{R}(P) \subset H_{0}^{1}(\mathcal{R})$, $\operatorname{dim} \mathrm{R}(P)=m$ we have that

$$
\mu_{i}=\max _{\substack{\mathcal{S} \subset \mathrm{R}(P), \operatorname{dim} \mathcal{S}=m-i+1}} \min _{\psi \in \mathcal{S}} \frac{\|\nabla \psi\|^{2}}{\|\psi\|^{2}}, \quad i=1, \ldots, m,
$$

are the Ritz values from $\mathrm{R}(P)$ and the Ritz vectors are $\psi_{i} \in \mathrm{R}(P), i=1, \ldots, m$ such that $\mu_{i}=\left\|\nabla \psi_{i}\right\|^{2},\left\|\psi_{i}\right\|^{2}=1$.

Let us now explain the estimation procedure on the example of the Dirichlet Laplace operator. Take $\psi \in \mathrm{R}(P) \subset H_{0}^{1}(\mathcal{R})$ and consider the solution $u(\psi)$ of the problem

$$
-\triangle u=\psi, \quad u \in H_{0}^{1}(\mathcal{R}) .
$$

Let the functions $u_{P}(\psi) \in \mathrm{R}(P), u_{1}\left(\psi, \mathcal{T}_{d}\right) \in \mathfrak{L}\left(\mathcal{T}_{d}\right)$, for $\psi \in \mathrm{R}(P)$, be such that

$$
\begin{aligned}
\left\|\nabla u(\psi)-\nabla u_{P}(\psi)\right\| & =\min _{v \in \mathrm{R}(P)}\|\nabla u(\psi)-\nabla v\|, \\
\left\|\nabla u(\psi)-\nabla u_{1}\left(\psi, \mathcal{T}_{d}\right)\right\| & =\min _{v \in \mathfrak{L}\left(\mathcal{T}_{d}\right)}\|\nabla u(\psi)-\nabla v\| .
\end{aligned}
$$

We define the approximation defects

$$
\begin{aligned}
\eta_{i}(P) & =\max _{\substack{\mathcal{S} \subset \mathrm{R}(P) \\
\operatorname{dim} \mathcal{S}=m-i+1}} \min _{\psi \in \mathcal{S}} \frac{\left\|\nabla u(\psi)-\nabla u_{P}(\psi)\right\|}{\|\nabla u(\psi)\|}, \\
\eta_{i}\left(P, \mathcal{T}_{d}\right) & =\max _{\substack{\mathcal{S} \subset \mathrm{R}(P) \\
\operatorname{dim} \mathcal{S}=m-i+1}} \min _{\psi \in \mathcal{S}} \frac{\left\|\nabla u(\psi)-\nabla u_{1}\left(\psi, \mathcal{T}_{d}\right)\right\|}{\|\nabla u(\psi)\|} .
\end{aligned}
$$

The quantities $\eta_{i}(P)$ are defined for any projection $P$ such that $\mathrm{R}(P) \subset H_{0}^{1}(\mathcal{R})$. They are the main ingredient of the error estimates below and we call them the approximation defects of $\mathrm{R}(P)$ or scaled residuals. Obviously, for $P_{d}$ from (2.8) we have $\eta_{i}\left(P_{d}\right)=\eta_{i}\left(P_{d}, \mathcal{T}_{d}\right)$ and we can profit from the information on the approximation properties of the spaces $\mathfrak{L}\left(\mathcal{T}_{d}\right)$ in the quest for obtaining computable estimates of $\eta_{i}\left(P_{d}\right)$. If the projection $P, \mathrm{R}(P) \subset \mathfrak{L}\left(\mathcal{T}_{d}\right)$ does not satisfy the assumption (2.8) we do not have the equality $\eta_{i}(P)=\eta_{i}\left(P, \mathcal{T}_{d}\right)$, but a simple perturbation argument can be used to obtain estimates of the approximation defect $\eta_{i}(P)$. We will comment on this more in Section 3 where we develop practical procedures for the computation of $\eta_{i}(P)$; cf. (3.11). The reason why we have chosen such test spaces 
is that we use an adaptation of the standard results on the hierarchical decomposition $\mathfrak{Q}\left(\mathcal{T}_{d}\right)=\mathfrak{L}\left(\mathcal{T}_{d}\right) \oplus \mathfrak{B}\left(\mathcal{T}_{d}\right)$ to obtain practical computational estimates for $\eta_{i}(P)$. This is the main subject of the following section.

The analysis of [19] yields the conclusion that the test space $\mathrm{R}(P)$ contains sufficiently good approximation for the eigenvalue $\lambda_{q}$ when $\eta_{m}(P)$ is smaller than half of the relative gap

$$
\gamma_{q}:=\min \left\{\frac{\lambda_{q+m}-\mu_{m}}{\lambda_{q+m}+\mu_{m}}, \frac{\mu_{1}-\lambda_{q-1}}{\mu_{1}+\lambda_{q-1}}\right\} .
$$

This is in line with the standard eigenvalue convergence analysis in Numerical Linear Algebra. We now state the results which follow by specializing the general results from [19, Theorems 3.3 and 3.4] and [19, Proposition 3.7] to the Dirichlet Laplace operator defined by (2.2) $-(2.3)$.

Theorem 2.1 (specialization of [19, Theorem 3.3] to $-\triangle$ ). Let $\lambda_{q-1}<\lambda_{q}=$ $\lambda_{q+m-1}<\lambda_{q+m}$ hold for the eigenvalues and let $\mathrm{R}(P) \subset H_{0}^{1}(\mathcal{R})$ be the test subspace such that $\operatorname{dim} \mathrm{R}(P)=m$ and $\frac{\eta_{m}(P)}{1-\eta_{m}(P)}<\gamma_{q}$. Then we have

$$
\left\|\operatorname{diag}\left(\frac{\left|\lambda_{q}-\mu_{i}\right|}{\mu_{i}}\right)_{i=1}^{m}\right\| \leq \frac{\eta_{m}(P)}{\mathfrak{g}_{q, \eta_{m}(P)}}\left\|\operatorname{diag}\left(\eta_{i}(P)\right)_{i=1}^{m}\right\|,
$$

where $\mathfrak{g}_{q, \zeta}:=\max \left\{\frac{\mu_{1}(1-\zeta)-\left(1+\frac{\zeta}{1-\zeta}\right) \lambda_{q-1}}{\left(1+\frac{\zeta}{1-\zeta}\right) \lambda_{q-1}}, \frac{\left(1-\frac{\zeta}{1-\zeta}\right) \lambda_{q+m}-(1+\zeta) \mu_{m}}{\left(1-\frac{\zeta}{1-\zeta}\right) \lambda_{q+m}}\right\}$ for $q>1$ and we set $\mathfrak{g}_{1, \zeta}:=\mathfrak{g}_{1}:=\frac{\lambda_{m+1}-\mu_{m}}{\lambda_{m+1}+\mu_{m}}$. Here we use $\operatorname{diag}\left(\alpha_{i}\right)_{i=1}^{m}$ to denote the $m \times m$ diagonal matrix with scalars $\alpha_{i}$ on its diagonal and $\|\cdot\|$ denotes any unitary invariant matrix norm and $\mu_{i}$ are the Ritz values from $\mathrm{R}(P)$.

In the case in which we do not have explicit information on the multiplicity of $\lambda_{q}$ we have a weaker upper estimate. To simplify the exposition we introduce the notation $\Sigma_{D}=\left\{\lambda_{i}: i \in \mathbb{N}\right\}$ and assume that we want to approximate the first $\mathrm{M} \in \mathbb{N}$ eigenvalues of (2.2). We now establish the equivalence of the error and the estimators $\eta_{i}$ in this case. This follows as an obvious combination of 19, Theorems 3.3 and 3.4] and [19, Proposition 3.7].

Theorem 2.2. Let $\lambda_{\mathrm{M}}<\lambda_{\mathrm{M}+1}$ and let $\lambda_{s_{1}}<\lambda_{s_{2}}<\cdots<\lambda_{s_{p}}$ be all the element 1 of $\left\{\lambda_{i}: i=1, \ldots, \mathrm{M}\right\}$. If $\frac{\eta_{\mathrm{M}}\left(L_{\mathrm{M}}(d)\right)}{1-\eta_{\mathrm{M}}\left(L_{\mathrm{M}}(d)\right)}<\frac{\lambda_{\mathrm{M}+1}-\lambda_{\mathrm{M}}\left(\mathcal{T}_{d}\right)}{\lambda_{\mathrm{M}+1}+\lambda_{\mathrm{M}}\left(\mathcal{T}_{d}\right)}$, then

$$
\frac{\lambda_{1}\left(\mathcal{T}_{d}\right)}{2 \lambda_{\mathrm{M}}\left(\mathcal{T}_{d}\right)} \sum_{i=1}^{\mathrm{M}} \eta_{i}^{2}\left(L_{\mathrm{M}}(d)\right) \leq \sum_{i=1}^{\mathrm{M}} \frac{\lambda_{i}\left(\mathcal{T}_{d}\right)-\lambda_{i}}{\lambda_{i}\left(\mathcal{T}_{d}\right)} \leq \frac{1}{\min _{i=1, \ldots, p} \mathfrak{g}_{s_{i}, \eta_{m_{i}}\left(P_{s_{i}}(d)\right)}} \sum_{i=1}^{\mathrm{M}} \eta_{i}^{2}\left(L_{\mathrm{M}}(d)\right) .
$$

Here $P_{s_{i}}(d)$ is the orthogonal projection onto the linear span of $\left\{u_{j}\left(\mathcal{T}_{d}\right): j=\right.$ $\left.\sum_{k=1}^{i} m_{k}+1, \ldots, \sum_{k=1}^{i+1} m_{k}\right\}$ and $m_{i}$ is the multiplicity of the eigenvalue $\lambda_{s_{i}}, i=$ $1, \ldots, p$. Obviously the identity $P_{s_{1}}(d) \oplus P_{s_{2}}(d) \oplus \cdots \oplus P_{s_{p}}(d)=L_{\mathrm{M}}(d)$ holds. In the case in which $\lambda_{1}=\lambda_{\mathrm{M}}$ we can drop the constant $\frac{\lambda_{1}\left(\mathcal{T}_{d}\right)}{2 \lambda_{M}\left(\mathcal{T}_{d}\right)}$ from the lower estimate.

Remark 2.3. Note that as $\eta_{m_{i}}\left(P_{s_{i}}(d)\right) \rightarrow 0$ we have

$$
\mathfrak{g}_{s_{i}, \eta_{m_{i}}\left(P_{s_{i}}(d)\right)} \rightarrow \min \left\{\frac{\lambda_{s_{i+1}}-\lambda_{s_{i}}}{\lambda_{s_{i}}}, \frac{\lambda_{s_{i}}-\lambda_{s_{i-1}}}{\lambda_{s_{i-1}}}\right\}
$$

\footnotetext{
${ }^{1}$ We assume that $1 \leq s_{1}<s_{2}<\cdots<s_{p} \leq \mathrm{M}$.
} 
and $\min _{i=1, \ldots, p} \mathfrak{g}_{s_{i}, \eta_{m_{i}}\left(P_{s_{i}}(d)\right)}$ quantifies the minimal relative gap among the eigenvalues $\lambda_{s_{1}}<\lambda_{s_{2}}<\cdots<\lambda_{s_{p}}$. Note that the relative gap $\mathfrak{g}_{s_{i}, \eta_{m_{i}}\left(P_{s_{i}}(d)\right)}$ distinguishes better between the close eigenvalues than the absolute gap, e.g. $\min \left\{\lambda_{s_{i+1}}-\right.$ $\left.\lambda_{s_{i}}, \lambda_{s_{i}}-\lambda_{s_{i-1}}\right\}$ is an example of an absolute gap. In Theorem 2.2, equivalently as in [13, Proposition 2.3], we have that when $\eta_{m_{i}}\left(P_{s_{i}}(d)\right)<\frac{1}{3} \min _{k \neq j} \frac{\left|\lambda_{s_{k}}-\lambda_{s_{j}}\right|}{\lambda_{s_{k}}+\lambda_{s_{j}}}$, $i=1, \ldots, p$, then

$$
\frac{1}{\min _{i=1, \ldots, p} \mathfrak{g}_{s_{i}, \eta_{m_{i}}\left(P_{s_{i}}(d)\right)}} \leq \frac{1}{\min _{k \neq j} \frac{\left|\lambda_{s_{k}}-\lambda_{s_{j}}\right|}{\lambda_{s_{k}}+\lambda_{s_{j}}}}
$$

Remark 2.4. The constant $\frac{\lambda_{1}\left(\mathcal{T}_{d}\right)}{2 \lambda_{M}\left(\mathcal{T}_{d}\right)}$ is not satisfactory, since it implies that the estimate is not quantitatively useful for higher eigenvalues. Establishing sharper lower estimate for higher eigenvalues is technically involved and does not promise any significant new quantitative information. Any type of estimate is bound to include the minimal relative gap between the computed Ritz values and the unwanted component of the spectrum, and estimating this distance is in practice only asymptotically possible. As an alternative we establish, in Section 4, an asymptotic exactness of the eigenvalue error and the approximation defects. This result holds for all discrete eigenvalues of a positive definite operator. It even holds for the eigenvalues which are in gaps of the essential spectrum (in case we are considering unbounded domains or periodic boundary conditions).

Let us note that Theorem 2.2 essentially solves the eigenvector approximation problem, too. By this we mean that we have both upper as well as lower estimates for the eigenvector error. This is made explicit in the following proposition.

Proposition 2.5. For eigenvectors $-\triangle v_{i}=\lambda_{i} v_{i},\left\|v_{i}\right\|=1$ there are Ritz vectors $\psi_{i}, \psi_{i} \in \mathrm{R}(P)$ and $\left\|\psi_{i}\right\|=1$ - assuming $\lambda_{q-1}<\lambda_{q} \leq \cdots \leq \lambda_{q+m-1}<\lambda_{q+m}$ and $2 \eta_{m}<\gamma_{q}-$ such that

$$
\begin{aligned}
\left\|v_{i}-\psi_{i}\right\| & \leq \max _{\lambda \in \Sigma_{D} \backslash\left\{\lambda_{i}\right\}} \frac{\sqrt{2 \lambda \mu_{i}}}{\left|\lambda-\mu_{i}\right|} \frac{\eta_{m}(P)}{\sqrt{1-\eta_{m}(P)}}, \\
\frac{\left\|\nabla \psi_{i}-\nabla v_{i}\right\|^{2}}{\left\|\nabla v_{i}\right\|^{2}} & =\left\|v_{i}-\psi_{i}\right\|^{2}+\frac{\mu_{i}-\lambda_{i}}{\lambda_{i}}, \quad i=q, \ldots, q+m-1 .
\end{aligned}
$$

The proof of (2.12) can be found in [17, Theorem 6.2] and identity (2.13) is well-known. We can now combine (2.13) with (2.12) and Theorem 2.2 to obtain equivalent estimators for the eigenvector error:

Corollary 2.6. Let the assumptions and the notation of Theorem 2.2 hold. Then there are eigenvectors $v_{i}$ and discrete eigenvectors $u_{i}\left(\mathcal{T}_{d}\right), i=1, \ldots, \mathrm{M}$ such that

$$
\frac{\lambda_{1}\left(\mathcal{T}_{d}\right)}{2 \lambda_{\mathrm{M}}\left(\mathcal{T}_{d}\right)} \sum_{i=1}^{\mathrm{M}} \eta_{i}^{2}\left(L_{\mathrm{M}}(d)\right) \leq \sum_{i=1}^{\mathrm{M}} \frac{\left\|\nabla u_{i}\left(\mathcal{T}_{d}\right)-\nabla v_{i}\right\|^{2}}{\left\|\nabla v_{i}\right\|^{2}} \leq \frac{3 \sum_{i=1}^{\mathrm{M}} \eta_{i}^{2}\left(L_{\mathrm{M}}(d)\right)}{\min _{i=1, \ldots, p} \mathfrak{g}_{s_{i}, \eta_{m_{i}}\left(P_{s_{i}}(d)\right)}} .
$$

This estimate can be both refined and generalized (to any positive definite operator) in an obvious way with the help of [19, Theorem 3.3 and Proposition 3.7] and [17, Theorem 6.2]. We leave out the details since their elaboration would not yield any new information. 


\section{Equivalence of the Eigenvalue/EigenVeCtor Estimator}

In the previous section, it was established that the approximation defects $\eta_{i}(P)$ are equivalent to their respective relative eigenvalue and eigenvector errors, and in Theorem 4.1 it will be shown that they actually provide asymptotically exact approximation of these relative errors. With this in mind, it is clear that approximation defects are useful theoretical tools in the analysis of eigenvalue/eigenvector approximations. That stated, they do not generally provide useful practical tools because they are not readily computed. In this section we consider computable estimates of the approximation defects $\eta_{i}(P)$ of $\mathrm{R}(P)$. In particular, we show that these estimates are equivalent to the approximation defects. We assume that the test subspace $\mathrm{R}(P)$ satisfies the same conditions as in (2.8), and for $\psi \in \mathrm{R}(P)$ we consider the functions $u_{1}\left(\psi, \mathcal{T}_{d}\right) \in \mathfrak{L}\left(\mathcal{T}_{d}\right), u_{2}\left(\psi, \mathcal{T}_{d}\right) \in \mathfrak{Q}\left(\mathcal{T}_{d}\right)$ and $\varepsilon\left(\psi, \mathcal{T}_{d}\right) \in \mathfrak{B}\left(\mathcal{T}_{d}\right)$ defined by:

$$
\begin{aligned}
\int_{\mathcal{R}} \nabla u_{1}\left(\psi, \mathcal{T}_{d}\right) \cdot \nabla v & =\int_{\mathcal{R}} \psi v \quad \text { for all } v \in \mathfrak{L}\left(\mathcal{T}_{d}\right), \\
\int_{\mathcal{R}} \nabla u_{2}\left(\psi, \mathcal{T}_{d}\right) \cdot \nabla v & =\int_{\mathcal{R}} \psi v \quad \text { for all } v \in \mathfrak{Q}\left(\mathcal{T}_{d}\right), \\
\int_{\mathcal{R}} \nabla \varepsilon\left(\psi, \mathcal{T}_{d}\right) \cdot \nabla v & =\int_{\mathcal{R}} \psi v-\nabla u_{1}\left(\psi, \mathcal{T}_{d}\right) \cdot \nabla v \quad \text { for all } v \in \mathfrak{B}\left(\mathcal{T}_{d}\right) .
\end{aligned}
$$

This definition for $u_{1}\left(\psi, \mathcal{T}_{d}\right)$ coincides with the minimization formulation given in Section 2, and $u_{2}\left(\psi, \mathcal{T}_{d}\right)$ satisfies the analagous minimization problem. The function $\varepsilon\left(\psi, \mathcal{T}_{d}\right)$ is the projection of the linear residual error onto the space of edge bump functions, and is an example of a hierarchical basis error estimator, which are well-known in the literature (see, for example, [2, Ch. 5] and [3]). The quantity $\left\|\nabla \varepsilon\left(\psi, \mathcal{T}_{d}\right)\right\|$ is much cheaper to compute than $\left\|\nabla u_{2}\left(\psi, \mathcal{T}_{d}\right)-\nabla u_{1}\left(\psi, \mathcal{T}_{d}\right)\right\|$, and provides a reliable estimate of the actual error $\left\|\nabla u(\psi)-\nabla u_{1}\left(\psi, \mathcal{T}_{d}\right)\right\|$.

Using arguments similar to those of Dörfler and Nochetto in [12, we will prove in Corollary 5.10 of Section 5 that there exists a constant $C_{1}\left(\mathcal{T}_{d}\right)$ depending solely on the shape regularity of $\mathcal{T}_{d}$ such that

$$
\left\|\nabla u(\psi)-\nabla u_{1}\left(\psi, \mathcal{T}_{d}\right)\right\| \leq C_{1}\left(\mathcal{T}_{d}\right)\left\|\nabla \varepsilon\left(\psi, \mathcal{T}_{d}\right)\right\|+\operatorname{osc}\left(\psi, \mathcal{T}_{d}\right) .
$$

The term $\operatorname{osc}\left(\psi, \mathcal{T}_{d}\right)$ is a measure of the oscillation in the data $\psi$. There are various ways of describing data oscillation to be found in the literature (see, for example, [12, 27]), and our definition will be similar in spirit to those. In Section 5, we will define $\operatorname{osc}\left(\psi, \mathcal{T}_{d}\right)$ explicitly and give a computable bound on $C_{1}\left(\mathcal{T}_{d}\right)$. For now, we merely state that, for $\psi \in \mathcal{H}_{0}^{1}(\mathcal{R})$,

$$
\operatorname{osc}\left(\psi, \mathcal{T}_{d}\right) \leq C_{2}\left(\mathcal{T}_{d}\right) d^{2}\|\nabla \psi\|
$$

where $C_{2}\left(\mathcal{T}_{d}\right)$ depends solely on the shape regularity of $\mathcal{T}_{d}$. Our estimate of $\eta_{i}(P)$ based on $\varepsilon\left(\psi, \mathcal{I}_{d}\right)$, for some $\mathrm{R}(P) \subset \mathfrak{L}\left(\mathcal{T}_{d}\right)$, is given by

$$
\eta_{i}\left(\mathfrak{B}_{d}, P\right)=\max _{\substack{\mathcal{S} \subset \mathrm{R}(P) \\ \operatorname{dim} \mathcal{S}=m-i+1}} \min _{\psi \in \mathcal{S}} \frac{\left\|\nabla \varepsilon\left(\psi, \mathcal{T}_{d}\right)\right\|}{\sqrt{\left\|\nabla u_{1}\left(\psi, \mathcal{T}_{d}\right)\right\|^{2}+\left\|\nabla \varepsilon\left(\psi, \mathcal{T}_{d}\right)\right\|^{2}}},
$$

and we have the following theorem.

Theorem 3.1. Let $-\triangle$ be the Dirichlet Laplacian in $\mathcal{R}$, which we triangulate with $\mathcal{T}_{d}$. If we take $P_{d}$ to be the orthogonal projection onto the linear span of $\psi_{i}^{d}$, 
$i=1, \ldots, m$ from (2.8), then

$$
1 \leq \frac{\eta_{i}\left(P_{d}\right)}{\eta_{i}\left(\mathfrak{B}_{d}, P_{d}\right)} \leq C_{1}\left(\mathcal{T}_{d}\right)+\max _{\psi \in \mathrm{R}\left(P_{d}\right),\|\psi\|=1} \frac{\operatorname{osc}\left(\psi, \mathcal{T}_{d}\right)}{\left\|\nabla \varepsilon\left(\psi, \mathcal{T}_{d}\right)\right\|},
$$

where $C_{1}\left(\mathcal{T}_{d}\right)$ is the optimal constant in (3.4).

Proof. Take an arbitrary $\psi \in \mathrm{R}\left(P_{d}\right)$. To establish the left-hand inequality, we first note that

$$
\begin{aligned}
\|\nabla u(\psi)\|^{2} & =\left\|\nabla\left(u(\psi)-u_{1}\left(\psi, \mathcal{T}_{d}\right)-\varepsilon\left(\psi, \mathcal{T}_{d}\right)\right)\right\|^{2}+\left\|\nabla u_{1}\left(\psi, \mathcal{T}_{d}\right)\right\|^{2}+\left\|\nabla \varepsilon\left(\psi, \mathcal{T}_{d}\right)\right\|^{2} \\
& \geq\left\|\nabla u_{1}\left(\psi, \mathcal{T}_{d}\right)\right\|^{2}+\left\|\nabla \varepsilon\left(\psi, \mathcal{T}_{d}\right)\right\|^{2}
\end{aligned}
$$

Therefore, we have

$$
\begin{aligned}
\frac{\left\|\nabla\left(u(\psi)-u_{1}\left(\psi, \mathcal{T}_{d}\right)\right)\right\|^{2}}{\|\nabla u(\psi)\|^{2}} & =1-\frac{\left\|\nabla u_{1}\left(\psi, \mathcal{T}_{d}\right)\right\|^{2}}{\|\nabla u(\psi)\|^{2}} \\
& \geq 1-\frac{\left\|\nabla u_{1}\left(\psi, \mathcal{T}_{d}\right)\right\|^{2}}{\left\|\nabla u_{1}\left(\psi, \mathcal{T}_{d}\right)\right\|^{2}+\left\|\nabla \varepsilon\left(\psi, \mathcal{T}_{d}\right)\right\|^{2}} \\
& =\frac{\left\|\nabla \varepsilon\left(\psi, \mathcal{T}_{d}\right)\right\|^{2}}{\left\|\nabla u_{1}\left(\psi, \mathcal{T}_{d}\right)\right\|^{2}+\left\|\nabla \varepsilon\left(\psi, \mathcal{T}_{d}\right)\right\|^{2}} .
\end{aligned}
$$

To prove the right-hand estimate, we note that

$$
\frac{\left\|\nabla u(\psi)-\nabla u_{1}\left(\psi, \mathcal{T}_{d}\right)\right\|}{\|\nabla u(\psi)\|} \leq C_{1}\left(\mathcal{T}_{d}\right) \frac{\left\|\nabla \varepsilon\left(\psi, \mathcal{T}_{d}\right)\right\|}{\|\nabla u(\psi)\|}+\frac{\operatorname{osc}\left(\psi, \mathcal{T}_{d}\right)}{\|\nabla u(\psi)\|} .
$$

Replacing $\|\nabla u(\psi)\|$ by $\sqrt{\left\|\nabla u_{1}\left(\psi, \mathcal{T}_{d}\right)\right\|^{2}+\left\|\nabla \varepsilon\left(\psi, \mathcal{T}_{d}\right)\right\|^{2}}$ in the denominator only increases the right-hand side. The conclusions of the theorem now follow readily from the definitions.

Under the standard nondegeneracy assumption,

$$
\left\|\nabla u(\psi)-\nabla u_{1}\left(\psi, \mathcal{T}_{d}\right)\right\| \sim d\|\psi\| \text { for sufficiently small } d,
$$

Theorem 3.1 establishes the equivalence of $\eta_{i}\left(P_{d}\right)$ and the computable $\eta_{i}\left(\mathfrak{B}_{d}, P_{d}\right)$. Here and elsewhere the notation $X \sim Y$ is used to indicate that, asymptotically, $c_{1} X \leq Y \leq c_{2} X$ for some constants $c_{1}, c_{2}>0$. In particular, the nondegeneracy assumption together with (3.4) and (3.5), imply that

$$
\operatorname{osc}\left(\psi, \mathcal{T}_{d}\right) \leq C_{2}\left(\mathcal{T}_{d}\right) \sqrt{\mu_{\max }\left(\mathrm{R}\left(P_{d}\right)\right)} d^{2}\|\psi\|
$$

and

$$
\left\|\nabla \varepsilon\left(\psi, \mathcal{T}_{d}\right)\right\| \sim\left\|\nabla u(\psi)-\nabla u_{1}\left(\psi, \mathcal{T}_{d}\right)\right\| \sim d\|\psi\|,
$$

where $\mu_{\max }\left(\mathrm{R}\left(P_{d}\right)\right)$ is the largest of the Ritz values associated with the orthonormal Ritz basis of $\mathrm{R}\left(P_{d}\right)$. Stated more explicitly and concisely, Theorem 3.1 together with (3.8), (3.9) and the fact that there is a constant $C \geq C\left(\mathcal{T}_{d}\right)$, yields

$$
1 \leq \lim _{d \rightarrow 0} \frac{\eta_{i}\left(P_{d}\right)}{\eta_{i}\left(\mathfrak{B}_{d}, P_{d}\right)} \leq C .
$$

Furthermore, the arguments in Section 5 provide a means of estimating the size of this constant directly from the shape regularity constraints on the geometry of the meshes.

The actual computation of the $\eta_{i}\left(\mathfrak{B}_{d}, P_{d}\right)$ involves solving a small, $m \times m$, generalized eigenvalue problem. Given a target range in which to look for eigenvalues 
of $-\Delta$ and a target number $m$ of Ritz values/vectors of the discretized operator to compute, the initial stage of computation returns Ritz values $\left\{\mu_{1}^{d}, \ldots, \mu_{m}^{d}\right\}$ and corresponding Ritz vectors $\left\{\psi_{1}^{d}, \ldots, \psi_{m}^{d}\right\}$; recall (2.8) from Section 2. It is these Ritz vectors that form the orthonormal basis for the space $\mathrm{R}\left(P_{d}\right)$ onto which $P_{d}$ projects. If we take $\varepsilon_{i}^{d}=\varepsilon\left(\psi_{i}^{d}, \mathcal{T}_{d}\right)$, it is clear that computing (3.6) for every $i$ is equivalent to solving the generalized eigenvalue problem

$$
E \mathbf{v}=\eta^{2}(E+D) \mathbf{v}, \quad D=\operatorname{diag}\left(\mu_{1}^{d}, \ldots, \mu_{m}^{d}\right), \quad E_{i j}=\int_{\mathcal{R}} \nabla \varepsilon_{i}^{d} \cdot \nabla \varepsilon_{j}^{d}
$$

Here we have relied on the fact that both $u_{1}\left(\psi, \mathcal{T}_{d}\right)$ and $\varepsilon\left(\psi, \mathcal{T}_{d}\right)$ depend on $\psi$ linearly.

We make a few final remarks before returning to consider the perturbation construction from Section 2 and what it tells us about the asymptotic behavior of the approximation defects. At the beginning of this section, we alluded to the fact that in Theorem 4.1 it is shown that

$$
\lim _{d \rightarrow 0} \frac{\sum_{i=1}^{m} \frac{\left|\mu_{i}^{d}-\lambda_{q}\right|}{\mu_{i}^{d}}}{\sum_{i=1}^{m} \eta_{i}^{2}\left(P_{d}\right)}=1 .
$$

In Section 6, we see experimentally that an analogous result to (3.12) appears to hold with $\eta_{i}\left(P_{d}\right)$ replaced by $\eta_{i}\left(\mathfrak{B}_{d}, P_{d}\right)$. This suggests that an even stronger result than asymptotic equivalence (3.10) may generally hold - namely

$$
\lim _{d \rightarrow 0} \frac{\eta_{i}\left(P_{d}\right)}{\eta_{i}\left(\mathfrak{B}_{d}, P_{d}\right)}=1
$$

Recent work by the second author [30] provides a partial explanation of why (3.13) could actually be expected in some situations. There it is shown that $\left\|\nabla \varepsilon\left(\psi, \mathcal{T}_{d}\right)\right\|$ is an asymptotically exact approximation of $\left\|\nabla\left(u(\psi)-u_{1}\left(\psi, \mathcal{I}_{d}\right)\right)\right\|$, provided that $u(\psi) \in H^{3}(\Omega) \cap W_{\infty}^{2}(\Omega)$ and certain approximate mesh symmetries are present throughout much of the mesh. Therefore, if these conditions hold for $\psi \in \mathrm{R}\left(P_{d}\right)$, we should expect behavior like (3.13). These smoothness assumptions are satisfied in our setting for convex domains, but not necessarily for nonconvex domains. To argue (3.13) properly would require a careful retracing and application of the arguments in [30. This is beyond the scope of the current paper, but we may revisit this idea in the future.

This same sort of reasoning suggests that error estimates based on gradient recovery might also work very well in this context; many such estimators have also been proven to yield asymptotically exact approximations of error under certain assumptions, and are seen to do so in practice even when these assumptions do not hold (or cannot be verified). For concreteness, we briefly mention the recovery scheme of Bank and Xu [5, 6] and how it can be used in our context. Given the piecewise constant $\nabla u_{1}\left(\psi, \mathcal{T}_{d}\right)$, each component of the gradient is $L^{2}$-projected into the space of continuous, piecewise-affine functions on $\mathcal{T}_{d}$ which do not vanish on the boundary, and then a few iterations of a multigrid-like smoother is applied to each component of the result. In symbols, we denote this, or any, gradient recovery 
procedure by

$$
\nabla u_{1}\left(\psi, \mathcal{T}_{d}\right) \mapsto \mathcal{G}_{d}\left(u_{1}\left(\psi, \mathcal{T}_{d}\right)\right)
$$

The corresponding analogue of our $\eta_{i}\left(\mathfrak{B}_{d}, P_{d}\right)$ is

$$
\eta_{i}\left(\mathcal{G}_{d}, P_{d}\right)=\max _{\substack{\mathcal{S} \subset R\left(P_{d}\right) \\ \operatorname{dim} \mathcal{S}=m-i+1}} \min _{\psi \in \mathcal{S}} \frac{\left\|\mathcal{G}_{d}\left(u_{1}\left(\psi, \mathcal{T}_{d}\right)\right)-\nabla u_{1}\left(\psi, \mathcal{T}_{d}\right)\right\|}{\left\|\mathcal{G}_{d}\left(u_{1}\left(\psi, \mathcal{T}_{d}\right)\right)\right\|} .
$$

Although we have only explicitly mentioned the Bank/Xu recovery scheme, others might also be used; but one should take care that the recovery scheme is linear with respect to $u_{1}\left(\psi, \mathcal{T}_{d}\right)$ so that the $\eta_{i}\left(\mathcal{G}_{d}, P_{d}\right)$ can be computed by solving a small generalized eigenvalue problem analogous to the one described above for the $\eta_{i}\left(\mathfrak{B}_{d}, P_{d}\right)$. In fact, many a posteriori error estimators could feasibly be used in this context; the main theoretical differences being with what we might be able to prove similar to Theorem 3.1

Finally, we remark briefly that, whatever procedure is used to compute $\psi_{i}^{d}$, we will in fact get a perturbation $\tilde{\psi}_{i}^{d}$ of it. However, the well-conditioning of the system associated with the computation of $\varepsilon_{i}(\psi)$-which we establish explicitly in Section [5-guarantees that this approximation error is not unduly magnified. In other words, the approximation of $E$ which we actually compute is of good quality and (3.11) is a well-behaved positive definite $m \times m$ generalized eigenvalue problem.

\section{On the asymptotic Behavior of the estimators $\eta_{i}\left(P_{d}\right)$}

Our analysis of the asymptotic properties of $\eta_{i}\left(P_{d}\right)$ is based on the abstract eigenvalue error representation result from [19, equation (3.9)]. Subsequently, most of the results from this section hold for any positive definite self-adjoint operator in a general Hilbert space, since this is the generality in which [19, equation (3.9)] has been proved. We will make this claim precise in the discussion at the end of this section. For now we concentrate on our model problem of the Dirichlet Laplace operator. This will reduce the notational burden on the reader, without sacrificing the generality of our technique.

For the form $h$ in (2.3) and some orthogonal projection $Y$, such that $\mathrm{R}(Y)=$ $\mathcal{Y} \subset H_{0}^{1}(\mathcal{R})$ and $\operatorname{dim} \mathcal{Y}<\infty$, we define the positive definite form $h_{Y}$, generically using $Y^{\perp}:=\mathbf{I}-Y$, by the formula

$$
h_{Y}(\psi, \phi)=h(Y \psi, Y \phi)+h\left(Y^{\perp} \psi, Y^{\perp} \phi\right), \quad \psi, \phi \in H_{0}^{1}(\mathcal{R}) .
$$

By $\mathbf{H}_{Y}$ we denote the self-adjoint operator which is defined by $h_{Y}$ in the sense of Kato (see 23, Theorem VI-2.23, p. 331]). We call $h_{Y}$ the block diagonal part of $h$ with respect to the decomposition $Y \oplus Y^{\perp}=\mathbf{I}$. Here we use $\mathbf{I}$ to denote the identity operator on $L^{2}(\mathcal{R})$. We also call $\mathbf{H}_{Y}$ the block diagonal part of the Dirichlet Laplace operator.

Let $Y_{d}$ be the orthogonal projection onto $\mathfrak{L}\left(\mathcal{T}_{d}\right)$ and let $P_{d}$ be as before. In this section we shall need the operators $\mathbf{H}_{P_{d}}$ and $\mathbf{H}_{Y_{d}}$, which are defined by the procedure (4.1). Some further properties of this construction can be found in [17, 18, 19] and the references therein. We collect those properties we shall need in the following list: 


$$
\begin{aligned}
h(\psi, \phi)-h_{P_{d}}(\psi, \phi) & =h\left(P_{d}^{\perp} \psi, P_{d} \phi\right)+h\left(P_{d} \psi, P_{d}^{\perp} \phi\right), \quad \psi, \phi \in H_{0}^{1}(\mathcal{R}), \\
h(\psi, \phi)-h_{Y_{d}}(\psi, \phi) & =h\left(Y_{d}^{\perp} \psi, Y_{d} \phi\right)+h\left(Y_{d} \psi, Y_{d}^{\perp} \phi\right), \quad \psi, \phi \in H_{0}^{1}(\mathcal{R}), \\
h_{Y_{d}}(\psi, \phi) & =h_{P_{d}}(\psi, \phi), \quad \phi \in \mathrm{R}\left(P_{d}\right), \psi \in H_{0}^{1}(\mathcal{R}), \\
h\left(Y_{d}^{\perp} \psi, Y_{d} \phi\right) & =h\left(P_{d}^{\perp} \psi, P_{d} \phi\right), \quad \phi \in \mathrm{R}\left(P_{d}\right), \psi \in H_{0}^{1}(\mathcal{R}), \\
\mathbf{H}_{P_{d}} \psi & =\mathbf{H}_{Y_{d}} \psi, \quad \psi \in \mathrm{R}\left(Y_{d}\right) \text { or } \psi \in \mathrm{R}\left(Y_{d}^{\perp}\right) \cap \mathcal{D}\left(\mathbf{H}_{Y_{d}}\right), \\
\eta_{m}\left(P_{d}\right) & =\max _{\substack{\psi, \phi \in H_{0}^{1}(\mathcal{R}) \\
\psi, \phi \neq 0}} \frac{\left|h(\psi, \phi)-h_{P_{d}}(\psi, \phi)\right|}{\sqrt{h_{P_{d}}(\psi, \psi) h_{P_{d}}(\phi, \phi)}} .
\end{aligned}
$$

We also need the following definitions. By $\Xi_{d}: \mathrm{R}\left(P_{d}\right) \rightarrow \mathrm{R}\left(P_{d}\right)$ and $\mathbf{W}_{d}: \mathrm{R}\left(P_{d}\right)^{\perp} \rightarrow$ $\mathrm{R}\left(P_{d}\right)^{\perp}$ we denote the operators which are defined by the form $h_{P_{d}}$-in the sense of Kato - in the spaces $\mathrm{R}\left(P_{d}\right)$ and $\mathrm{R}\left(P_{d}\right)^{\perp}$, respectively. The operator $\Xi_{d}$ is called the (generalized) Rayleigh quotient. Note that $\Xi_{d} \psi_{i}^{d}=\mu_{i}^{d} \psi_{i}^{d},\left\|\psi_{i}^{d}\right\|=1$ holds for the Ritz values $\mu_{i}^{d}$ and Ritz vectors $\psi_{i}^{d}$ from (2.8).

To study the asymptotic behavior of $\eta_{i}\left(P_{d}\right)$ we make the following standard convergence assumption (cf. [21, Assumption (2.14)]),

$$
\left\|\nabla u(\psi)-\nabla u_{1}\left(\psi, \mathcal{T}_{d}\right)\right\| \leq \mathfrak{C} d^{\alpha_{1}}\|\nabla \psi\|, \quad \psi \in H_{0}^{1}(\mathcal{R})
$$

and $\mathfrak{C}, \alpha_{1}, 0<\alpha_{1}$ are independent of $d$ and $\psi$. We also make an abstract nondegeneracy assumption (similar in spirit to (3.7)),

$$
\left\|\nabla u(\psi)-\nabla u_{P_{d}}(\psi)\right\| \geq \mathfrak{c} d^{\alpha_{1}}\|\nabla \psi\|, \quad \psi \in \mathrm{R}\left(P_{d}\right) .
$$

The constant $\mathfrak{c}$ is naturally also assumed to be independent from $d$ and $\psi$. If there exists a disc $\mathfrak{D} \subset \mathcal{R}$ and a constant $k>0$ such that

$$
\min \left\{\operatorname{diam}(T): T \in \mathcal{T}_{d} \text { and } T \subset \mathfrak{D}\right\} \geq k d,
$$

then according to [14, Remark 4.1] the assumption (4.3) holds.

Without reducing the level of generality we may assume that we are given an eigenvalue $\lambda_{q}$ of multiplicity $m$ and Ritz vectors $\psi_{i}^{d}$ which are paired with eigenvectors $v_{i},\left\|v_{i}\right\|=1, i=1, \ldots, m$ in the sense of Proposition 2.5. cf. 17. Theorem 6.2]. The general case of a cluster of eigenvalues $\lambda_{q} \leq \lambda_{q+1} \leq \cdots \leq \lambda_{q+m-1}$ can be reduced to the case of a single multiple eigenvalue and the conclusions, identities (4.4) and (4.5) below, remain unchanged.

Theorem 4.1. Let the assumptions (4.2)-(4.3) hold and let $P_{d}$ from (2.8) be such that $\frac{\eta_{m}\left(P_{d}\right)}{1-\eta_{m}\left(P_{d}\right)}<\gamma_{q}^{d}:=\min \left\{\frac{\lambda_{q+m}-\mu_{m}^{d}}{\lambda_{q+m}+\mu_{m}^{d}}, \frac{\mu_{1}^{d}-\lambda_{q-1}}{\mu_{1}^{d}+\lambda_{q-1}}\right\}$ for all $d$. Then, assuming the pairing of eigenvectors and Ritz vectors as before, we have

$$
\begin{aligned}
& \lim _{d \rightarrow 0} \frac{\sum_{i=1}^{m} \frac{\left|\mu_{i}^{d}-\lambda_{q}\right|}{\mu_{i}^{d}}}{\eta_{1}^{2}\left(P_{d}\right)+\cdots+\eta_{m}^{2}\left(P_{d}\right)}=1, \\
& \lim _{d \rightarrow 0} \frac{\sum_{i=1}^{m} \frac{\left\|\nabla \psi_{i}^{d}-\nabla v_{i}\right\|^{2}}{\left\|\nabla v_{i}\right\|^{2}}}{\eta_{1}^{2}\left(P_{d}\right)+\cdots+\eta_{m}^{2}\left(P_{d}\right)}=1 .
\end{aligned}
$$

Analogous asymptotic properties are shared by other measures of the relative error from (2.11). 
Proof. The bounded symmetric form $h\left(\mathbf{H}_{P_{d}}^{-1 / 2} \cdot, \mathbf{H}_{P_{d}}^{-1 / 2} \cdot\right)-\lambda_{q}\left(\mathbf{H}_{P_{d}}^{-1 / 2} \cdot, \mathbf{H}_{P_{d}}^{-1 / 2} \cdot\right)$ can be represented - with respect to $P_{d} \oplus P_{d}^{\perp}=\mathbf{I}$ - by the bounded operator matrix

$$
H_{s}\left(\lambda_{q}\right)=\left[\begin{array}{cc}
\mathbf{I}-\lambda_{q} \Xi_{d}^{-1} & \Gamma_{d}^{*} \\
\Gamma_{d} & \mathbf{I}-\lambda_{q} \mathbf{W}_{d}^{-1}
\end{array}\right], \quad \text { and } \Gamma_{d}: \mathrm{R}\left(P_{d}\right) \rightarrow \mathrm{R}\left(P_{d}^{\perp}\right)
$$

is such that $\left(\psi, \Gamma_{d} \phi\right)=h\left(\mathbf{H}_{P_{d}}^{-1 / 2} \psi, \mathbf{H}_{P_{d}}^{-1 / 2} \phi\right)-h_{P_{d}}\left(\mathbf{H}_{P_{d}}^{-1 / 2} \psi, \mathbf{H}_{P_{d}}^{-1 / 2} \phi\right), \psi \in \mathrm{R}\left(P_{d}^{\perp}\right)$, $\phi \in \mathrm{R}\left(P_{d}\right)$ holds. The explicit formula for $\Gamma_{d}$ can be extracted from [17, equation (4.37)]. Using standard Schur complement Wilkinson's tricks (from Numerical Linear Algebra) on the identity in (4.6) we can conclude - as has been done in [19, equation (3.9)] - that

$$
\mathbf{I}-\lambda_{q} \Xi_{d}^{-1}=\Gamma_{d}^{*}\left(\mathbf{I}-\lambda_{q} \mathbf{W}_{d}^{-1}\right)^{-1} \Gamma_{d} .
$$

This error representation formula is the basis for our argument. Before we proceed, note that $\operatorname{tr}\left(\mathbf{I}-\lambda_{q} \Xi_{d}^{-1}\right)=\sum_{i=1}^{m} \frac{\mu_{i}^{d}-\lambda_{i}}{\mu_{i}^{d}}$ and that $\operatorname{tr}\left(\Gamma_{d}^{*} \Gamma_{d}\right)=\eta_{1}\left(P_{d}\right)^{2}+\cdots+\eta_{m}^{2}\left(P_{d}\right)$. In particular, we have the following characterization: after setting $\mathcal{Q}:=H_{0}^{1}(\mathcal{R})$,

$$
\begin{aligned}
& \left\|\Gamma_{d}\right\|=\eta_{m}\left(P_{d}\right)=\max _{\substack{\psi, \phi \in \mathcal{Q} \\
\psi, \phi \neq 0}} \frac{\left|h(\psi, \phi)-h_{P_{d}}(\psi, \phi)\right|}{\sqrt{h_{P_{d}}(\psi, \psi) h_{P_{d}}(\phi, \phi)}} \\
& =\max _{\substack{\psi, \phi \in \mathcal{Q} \\
\psi, \phi \neq 0}} \frac{\left|h(\psi, \phi)-h_{P_{d}}(\psi, \phi)\right|}{\left\|\mathbf{H}_{P_{d}}^{1 / 2} \psi\right\|\left\|\mathbf{H}_{P_{d}}^{1 / 2} \psi\right\|}=\max _{\substack{\psi \in \mathcal{Q}, \phi \in R \\
\psi, \phi \neq 0}} \frac{\left|h(\psi, \phi)-h_{P_{d}}(\psi, \phi)\right|}{\left\|\mathbf{H}_{P_{d}}^{1 / 2} \psi\right\|\left\|\mathbf{H}_{P_{d}}^{1 / 2} \psi\right\|} \\
& =\max _{\substack{\psi \in \mathcal{Q}, \phi \in R\left(P_{d}\right) \\
\psi, \phi \neq 0}} \frac{\left|h(\psi, \phi)-h_{Y_{d}}(\psi, \phi)\right|}{\left\|\mathbf{H}_{P_{d}}^{1 / 2} \psi\right\|\left\|\mathbf{H}_{P_{d}}^{1 / 2} \phi\right\|}=\max _{\substack{\psi \in \mathcal{Q}, \phi \in R\left(P_{d}\right) \\
\psi, \phi \neq 0}} \frac{\left|h\left(Y_{d}^{\perp} \psi, Y_{d} \phi\right)\right|}{\left\|\mathbf{H}_{P_{d}}^{1 / 2} \psi\right\|\left\|\mathbf{H}_{P_{d}}^{1 / 2} \phi\right\|} \\
& =\max _{\substack{\psi \in \mathcal{Q}, \phi \in R\left(P_{d}\right) \\
\psi, \phi \neq 0}} \frac{\sqrt{h\left[Y_{d}^{\perp} \psi\right] h\left[Y_{d} \phi\right]}}{\left\|\mathbf{H}_{P_{d}}^{1 / 2} \psi\right\|\left\|\mathbf{H}_{P_{d}}^{1 / 2} \phi\right\|} \leq \max _{\substack{\psi \in \mathcal{Q}, \phi \in R\left(P_{d}\right) \\
\psi, \phi \neq 0}} \frac{\sqrt{h\left[Y_{d}^{\perp} \psi\right] h\left[Y_{d} \phi\right]}}{\left\|\mathbf{H}_{P_{d}}^{1 / 2} \psi\right\|\left\|\mathbf{H}_{P_{d}}^{1 / 2} \phi\right\|} \\
& \leq \frac{\mathfrak{C}_{1}}{\sqrt{1-\eta_{m}\left(P_{d}\right)}} d^{\alpha_{1}}
\end{aligned}
$$

We can write (4.7) as

$$
\mathbf{I}-\lambda_{q} \Xi_{d}^{-1}=\Gamma_{d}^{*} \Gamma_{d}+\lambda_{q} \Gamma_{d}^{*} \mathbf{W}_{d}^{-1 / 2}\left(\mathbf{I}-\lambda_{q} \mathbf{W}_{d}^{-1}\right)^{-1} \mathbf{W}_{d}^{-1 / 2} \Gamma_{d}
$$

Note that $\min \left\{\frac{\lambda_{1}}{\lambda_{q}-\lambda_{1}}, 1\right\} \leq \nu \leq \mathfrak{g}_{q, \eta_{m}\left(P_{i}\right)}$, for all $\nu \in \Sigma\left(\left|\left(\mathbf{I}-\lambda_{q} \mathbf{W}_{d}^{-1}\right)^{-1}\right|\right)$, and so asymptotically it is sufficient to analyze $s_{i}\left(\mathbf{W}^{-1 / 2} \Gamma_{d}\right), i=1, \ldots, m$, i.e. the singular values of $\mathbf{W}^{-1 / 2} \Gamma_{d}$. As in (4.8), the estimate

$$
\begin{aligned}
\left\|\mathbf{W}_{d}^{-1 / 2} \Gamma_{d}\right\| & =\max _{\substack{\psi \in \mathcal{D}\left(\mathbf{H}_{P_{d}}\right), \phi \in \mathrm{R}\left(P_{d}\right) \\
\psi, \phi \neq 0}} \frac{\left|h(\psi, \phi)-h_{P_{d}}(\psi, \phi)\right|}{\left\|\mathbf{H}_{P_{d}} \psi\right\|\left\|\mathbf{H}_{P_{d}}^{1 / 2} \phi\right\|} \\
& =\max _{\substack{\psi \in \mathcal{D}\left(\mathbf{H}_{P_{d}}\right), \phi \in \mathrm{R}\left(P_{d}\right) \\
\psi, \phi \neq 0}} \frac{\left|h\left(Y_{d}^{\perp} \psi, Y_{d} \phi\right)\right|}{\left\|\mathbf{H}_{P_{d}} \psi\right\|\left\|\mathbf{H}_{P_{d}}^{1 / 2} \phi\right\|} \\
& \leq \max _{\substack{\psi \in \mathrm{R}\left(Y_{d}\right)^{\perp}, \psi \in \mathcal{D}\left(\mathbf{H}_{P_{d}}\right), \phi \in \mathrm{R}\left(P_{d}\right) \\
\psi, \phi \neq 0}} \frac{\sqrt{h\left[Y_{d}^{\perp} \psi\right] h\left[Y_{d} \phi\right]}}{\left\|\mathbf{H}_{Y_{d}} \psi\right\|\left\|\mathbf{H}_{Y_{d}}^{1 / 2} \phi\right\|} \\
& \leq \eta_{m}\left(P_{d}\right)\left\|\mathbf{H}_{Y_{d}}^{-1 / 2} Y_{d}^{\perp}\right\|
\end{aligned}
$$


holds. By an analogous computation we obtain

$$
\left\|\mathbf{W}_{d}^{-1 / 2} \Gamma_{d} \phi\right\| \leq\left\|\mathbf{H}_{Y_{d}}^{-1 / 2} Y_{d}^{\perp}\right\|\left\|\Gamma_{d} \phi\right\|, \quad \phi \in \mathrm{R}\left(P_{d}\right),
$$

which yields the estimate

$$
s_{i}\left(\mathbf{W}_{d}^{-1 / 2} \Gamma_{d}\right) \leq \eta_{i}\left(P_{d}\right)\left\|\mathbf{H}_{Y_{d}}^{-1 / 2} Y_{d}^{\perp}\right\|, \quad i=1, \ldots, m .
$$

Let us now combine the assumptions (4.2) and (4.3) with (4.8) and (4.10). Assumptions (4.2) and (4.3) and the characterization(4.8) imply $\operatorname{tr}\left(\Gamma_{d}^{*} \Gamma_{d}\right)=O\left(d^{2 \alpha_{1}}\right)$. Furthermore, since $\lim _{d \rightarrow 0}\left\|\mathbf{H}_{Y_{d}}^{-1 / 2} Y_{d}^{\perp}\right\| \rightarrow 0$, we conclude that

$$
\lim _{d \rightarrow 0} \frac{\operatorname{tr}\left(\Gamma_{d}^{*} \mathbf{W}_{d}^{-1 / 2}\left(\mathbf{I}-\lambda_{q} \mathbf{W}_{d}^{-1}\right)^{-1} \mathbf{W}_{d}^{-1 / 2} \Gamma_{d}\right)}{\operatorname{tr}\left(\Gamma_{d}^{*} \Gamma_{d}\right)}=0 .
$$

If we now apply the trace operator $\operatorname{tr}(\cdot)$ on the equation (4.9) and utilize (4.10)(4.11) we obtain the conclusion (4.4). The eigenvector estimate follows with the help of Proposition 2.5

4.1. Remarks on possible extensions. Let us summarize the assumptions which have been made on the way to Theorem 4.1 and examine their importance. We assume that we have the environment Hilbert space $\mathcal{H}$ with the norm $\|\cdot\|$ and the scalar product $(\cdot, \cdot)$. We need a positive definite (in $\mathcal{H}$ ) and closed symmetric form $h$ and two sequences of orthogonal projections $P_{d}$ and $Y_{d}$ with the properties to be specified below.

As has already been mentioned, according to [23, Theorem VI-2.23, p. 331] a general positive definite symmetric form $h$ with the domain of definition $\mathcal{Q}(h)$ defines in $\mathcal{H}$ the self-adjoint operator $\mathbf{H}$ such that $\mathcal{Q}(h)=\mathcal{D}\left(\mathbf{H}^{1 / 2}\right)$ and $h(\psi, \phi)=$ $\left(\mathbf{H}^{1 / 2} \psi, \mathbf{H}^{1 / 2} \phi\right), \psi, \phi \in \mathcal{Q}(h)$. Let $\lambda_{q}$ be a discrete eigenvalue of the operator $\mathbf{H}$ of multiplicity $m$ and let $E_{q}$ be the orthogonal projection onto the eigenspace of $\lambda_{q}$. We assume that $Y_{d}$ is a sequence of orthogonal projections such that $\mathrm{R}\left(Y_{d}\right) \subset \mathcal{Q}(h)$, $\operatorname{dim} \mathrm{R}\left(Y_{d}\right)<\infty$ and $Y_{d} \rightarrow \mathbf{I}$ strongly. We use a straightforward modification of (4.1) to define the self-adjoint operators $\mathbf{H}_{Y_{d}}$. For details see [17, 19.

Assumptions. Now let $P_{d}$ be a sequence of orthogonal projections such that $\mathrm{R}\left(P_{d}\right) \subset \mathrm{R}\left(Y_{d}\right), \operatorname{dim} \mathrm{R}\left(P_{d}\right)=m, P_{d}$ commutes with $\mathbf{H}_{Y_{d}}$ and $\left\|E_{q}-P_{d}\right\| \rightarrow 0$. Also let $\mathbf{H}_{P_{d}}$ and $\Xi_{d}$ be the operators defined by the procedure (4.1) and let $\mu_{i}^{d}$ be the eigenvalues of $\Xi_{d}$.

Conclusion. Then, under the abstract convergence and nondegeneracy assumptions, where $\|\cdot\|_{\mathbf{H}}=\left\|\mathbf{H}^{1 / 2} \cdot\right\|$ is the energy norm,

$$
\begin{aligned}
& \left\|\mathbf{H}^{-1} \psi-\mathbf{H}_{Y_{d}}^{-1} \psi\right\|_{\mathbf{H}} \leq \mathfrak{C} d^{\alpha_{1}}\|\psi\|_{\mathbf{H}}, \quad \psi \in \mathcal{Q}(h), \\
& \left\|\mathbf{H}^{-1} \psi-\mathbf{H}_{P_{d}}^{-1} \psi\right\|_{\mathbf{H}} \geq \mathfrak{c} d^{\alpha_{1}}\|\psi\|_{\mathbf{H}}, \quad \psi \in \mathrm{R}\left(P_{d}\right),
\end{aligned}
$$

we have

$$
\lim _{d \rightarrow 0} \frac{\sum_{i=1}^{m} \frac{\left|\mu_{i}^{d}-\lambda_{q}\right|}{\mu_{i}^{d}}}{\eta_{1}^{2}\left(P_{d}\right)+\cdots+\eta_{m}^{2}\left(P_{d}\right)}=1 .
$$

The proof is a verbatim reformulation of the proof of Theorem 4.1 and we omit it. The constants $\alpha_{1}, \mathfrak{c}$ and $\mathfrak{C}$ are assumed to be independent of $d$ and $\psi$ and we call $\mu_{i}^{d}$ the abstract Ritz values. 
The example of the Dirichlet Laplace operator serves to show that the assumptions (4.12)-(4.13) are plausible. In fact, the asymptotic assumptions (4.12)-(4.13) are only made for technical convenience. The conclusion (4.14) follows under much milder assumptions. In fact, in the proof of Theorem 4.1 we see that to prove (4.14) it is sufficient to assume that $\frac{\eta_{m}\left(P_{d}\right)}{1-\eta_{m}\left(P_{d}\right)} \leq \widetilde{\gamma}_{d}:=\min \left\{\frac{\lambda_{\text {lar }}(\mathbf{H})-\mu_{m}^{d}}{\lambda_{\operatorname{lar}}(\mathbf{H})+\mu_{m}^{d}}, \frac{\mu_{1}^{d}-\lambda_{\text {smal }}(\mathbf{H})}{\mu_{1}^{d}+\lambda_{\text {smal }}(\mathbf{H})}\right\}$, $\eta_{m}\left(P_{d}\right) \rightarrow 0$ as $d \rightarrow 0$ and $\eta_{1}\left(P_{d}\right)>0$, for $d>0$. Here we have used $\lambda_{\text {smal }}(\mathbf{H})$ and $\lambda_{\text {lar }}(\mathbf{H})$, such that $\lambda_{\text {smal }}(\mathbf{H})<\lambda_{q}<\lambda_{\text {lar }}(\mathbf{H})$ to denote the nearest points in $\Sigma(\mathbf{H}) \backslash\left\{\lambda_{q}\right\}$ to $\lambda_{q}$. In the case in which $\eta_{1}\left(P_{d}\right)=0$ for some $d>0$ we have that $v \in \mathrm{R}\left(P_{d}\right)$, for $v$ an eigenvector of the operator $\mathbf{H}$. This case can be considered as trivial and excluded without reducing the level of generality. The assumptions, (4.2) - 4.3 - and their abstract variants (4.12)-4.13) - are, however, typically satisfied by most approximation methods; cf. [14, Remark 4.1]. Also note that 20, Theorem 3.3] yields that the assumptions $\frac{\eta_{m}\left(P_{d}\right)}{1-\eta_{m}\left(P_{d}\right)} \leq \widetilde{\gamma}_{d}$, for all $d$ and $\eta_{m}\left(P_{d}\right) \rightarrow 0$ as $d \rightarrow 0$ imply

$$
\left\|P_{d}-E_{q}\right\| \leq \frac{4}{\widetilde{\gamma}_{d}} \frac{\eta_{m}\left(P_{d}\right)}{\sqrt{1-\eta_{m}\left(P_{d}\right)}} \rightarrow 0
$$

so we do not have to assume $\left\|P_{d}-E_{q}\right\| \rightarrow 0$ explicitly.

This shows that the asymptotic exactness of our "ideal" estimators follows from simple algebraic properties of the positive definite form $h$ only. Given the abstract nondegeneracy assumption, we establish our proof without any reference to finer properties of the structure of the form $h$. We only use its positive definiteness. This gives us confidence that we shall be able to apply this framework to more general spectral problems with certain ease. This will be a subject of subsequent reports.

\section{Concerning CONStants And COMPUtational COST}

In this section we discuss what we might reasonably expect from the constant $C_{1}\left(\mathcal{T}_{d}\right)$ appearing in Theorem 3.1 , and give a sense of the cost of computing the bump function error estimators by providing some bounds on the condition number and spectral radius of the corresponding linear system. All of these quantities of interest depend only on the underlying triangulation $\mathcal{T}_{d}$, so it is natural to discuss them together. We will make use of the following identities.

Lemma 5.1. Let $\tau \in \mathcal{T}_{d}$ be given and let $z_{k}, x_{k}, \theta_{k}, e_{k}, L_{k}, h_{k}, \ell_{k}$ and $b_{k}, k=1,2,3$, denote, respectively: vertex $k$, the coordinates of $z_{k}$, the measure of the associated interior angle, the edge opposite $z_{k}$, the length of $e_{k}$, the distance from $z_{k}$ to the line containing $e_{k}$, the linear basis function associated with $z_{k}$ and the quadratic bubble basis function associated with $e_{k}$. Furthermore, let $p, q, r \in \mathbb{Z}_{\geq 0}$. The following hold:

$$
\begin{aligned}
& \int_{\tau} \ell_{1}^{p} \ell_{2}^{q} \ell_{3}^{r}=\frac{p ! q ! r !}{(p+q+r+2) !} 2|\tau|, \quad \int_{e_{k}} \ell_{k-1}^{p} \ell_{k+1}^{q}=\frac{p ! q !}{(p+q+1) !} L_{k}, \\
& \left\|\nabla \ell_{k}\right\|_{\tau}^{2}=\frac{1}{2}\left(\cot \theta_{k-1}+\cot \theta_{k+1}\right), \quad \int_{\tau} \nabla \ell_{k-1} \cdot \nabla \ell_{k+1}=-\frac{1}{2} \cot \theta_{k}, \\
& \left\|\nabla b_{k}\right\|_{\tau}^{2}=\frac{4}{3}\left(\cot \theta_{1}+\cot \theta_{2}+\cot \theta_{3}\right), \quad \int_{\tau} \nabla b_{k-1} \cdot \nabla b_{k+1}=-\frac{4}{3} \cot \theta_{k},
\end{aligned}
$$




$$
\begin{aligned}
& \nabla \ell_{k} \cdot\left(x-x_{k-1}\right)=\nabla \ell_{k} \cdot\left(x-x_{k+1}\right)=\ell_{k}, \\
& \left\|x-x_{k}\right\|_{\tau}^{2}=\frac{\cot \theta_{k-1}+3 \cot \theta_{k}+\cot \theta_{k+1}}{3}|\tau|^{2}, \\
& \text { For } f \in H^{1}(\tau), h_{k} \int_{e_{k}} f=\int_{\tau} 2 f+\left(x-x_{k}\right) \cdot \nabla f .
\end{aligned}
$$

Most, if not all, of these identities are well-known, and (5.1)-(5.5) can be verified by direct computation; integration by parts yields (5.6). We will also use

$$
g\left(z_{k}, \tau\right):=\left\|x-x_{k}\right\|_{\tau} /|\tau|
$$

in what follows.

5.1. A more careful look at (3.4). A derivation of (3.4) which will give us fairly detailed information on the constants involved and the data oscillation will require a careful look at some Clément-like quasi-interpolation estimates and the key arguments of Dörfler and Nochetto in [12. For the arguments that follow, we consider a fixed $\psi \in L^{2}(\mathcal{R})$; this is certainly more general than we need for the results in Section 3, but the arguments given below do apply in more general circumstances. To make the notation less cumbersome, we will generally suppress explicit dependencies on $\psi$ and $\mathcal{T}_{d}$. For any $v \in H_{0}^{1}(\mathcal{R})$ and any $\mathcal{I} v \in \mathfrak{L}\left(\mathcal{T}_{d}\right)$, we have the well-known identity

$$
\int_{\mathcal{R}} \nabla\left(u-u_{1}\right) \cdot \nabla v=\int_{\mathcal{R}} \psi(v-\mathcal{I} v)-\nabla u_{1} \cdot \nabla(v-\mathcal{I} v) .
$$

We aim to bound this in terms of $\|\nabla \varepsilon\|$ and $\|\nabla v\|$, the data oscillation osc $(\psi)-$ which will be defined later - and constants which depend only on the shape regularity of the mesh.

For the analysis below, we take $\mathcal{I} v$ to be the modified version of the Clément interpolant which is identical to that introduced by Carstensen [9], except near the boundary. Our analysis is partially motivated by that in $[9,12,28,35$, and is quite similar at some points to that in 28. It will be convenient for us to consider the set $\overline{\mathcal{V}}$ of all vertices in $\mathcal{T}_{d}$, including those on $\partial \mathcal{R}$. For some of the lemmas it will also be convenient to distinguish the set of all non-Dirichlet edges which have $z \in \overline{\mathcal{V}}$ as a vertex; we denote this set of edges $\mathcal{E}_{z}$.

Recall that $\ell_{z}$ is the continuous, piecewise linear function such that $\ell_{z}(z)=1$ and $\ell_{z}\left(z^{\prime}\right)=0$ for $z^{\prime} \in \overline{\mathcal{V}} \backslash\{z\}$. We take $\omega_{z}$ to be the support of $\ell_{z}$. In what follows, for $f \in L^{2}(\mathcal{R})$, we define

$$
f_{z}=\left(\int_{\omega_{z}} f \ell_{z}\right) /\left(\int_{\omega_{z}} \ell_{z}\right), \quad \mathcal{I} f=\sum_{z \in \mathcal{V}} f_{z} \ell_{z} .
$$

The key properties of this interpolant are that,

$$
\int_{\mathcal{R}} \nabla u_{1} \cdot \nabla(v-\mathcal{I} v)=\sum_{z \in \mathcal{V}} \int_{\omega_{z}} \nabla u_{1} \cdot \nabla\left[\left(v-v_{z}\right) \ell_{z}\right]+\sum_{z \in \overline{\mathcal{V}} \backslash \mathcal{V}} \int_{\omega_{z}} \nabla u_{1} \cdot \nabla\left(v \ell_{z}\right) .
$$


These identities follow directly from

$$
\sum_{z \in \overline{\mathcal{V}}} \ell_{z}=1 \text { on } \mathcal{R}, \quad \int_{\omega_{z}}\left(v-v_{z}\right) \ell_{z}=0 .
$$

We first treat (5.9), and to do so we will use the following lemma.

Lemma 5.2. Let $f \in H_{0}^{1}(\mathcal{R})$. The following hold:

$$
\begin{aligned}
& \text { For } z \in \overline{\mathcal{V}}, ;\left\|\left(f-f_{z}\right) \ell_{z}^{1 / 2}\right\|_{\omega_{z}}^{2} \leq \frac{D_{z}^{2}}{\pi^{2}}\|\nabla f\|_{\omega_{z}}^{2}, \\
& \text { For } z \in \overline{\mathcal{V}} \backslash \mathcal{V},\|f\|_{\omega_{z}}^{2} \leq \kappa_{z}^{2}\|\nabla f\|_{\omega_{z}}^{2}
\end{aligned}
$$

where $D_{z}=\operatorname{diam}\left(\omega_{z}\right)$ and $\kappa_{z}^{2}$, given explicitly below, scales like $\left|\omega_{z}\right|$.

Proof. The first inequality is a direct application of recent work by Chua and Wheeden [11, Theorems 1.1 and 1.2] on weighted Poincaré inequalities. Their work extends previous contributions by Payne and Weinberger, Acosta and Durán, and Bebendorf [1, 8, 31. Although the results in [11 are stated for convex domains, we need not be concerned here with whether or not $\omega_{z}$ is convex, because the weight functions $\ell_{z}$ are supported in $\omega_{z}$.

To establish the second inequality, we take $\alpha<\beta$ and the Lipschitz function $\rho(\theta)$ such that $\omega_{z}=\{z+t(\cos \theta, \sin \theta) \mid 0 \leq t \leq \rho(\theta), \alpha \leq \theta \leq \beta\}$. Using the Poincaré-Friedrichs' estimate in [10, Theorem 3.1], we choose

$$
\kappa_{z}^{2}=2\left|\omega_{z}\right| \int_{\alpha}^{\beta} 1+\left(\frac{\rho^{\prime}(\theta)}{\rho(\theta)}\right)^{2} d \theta .
$$

Recognizing that the integral is scale-invariant completes the proof.

We are now ready to provide a bound for $\left|\int_{\mathcal{R}} \psi(v-\mathcal{I} v)\right|$ in terms of $\|\nabla v\|$ and our first definition of oscillation of $\psi$.

Theorem 5.3. For $v \in H_{0}^{1}(\mathcal{R})$, it holds that

$$
\begin{aligned}
\left|\int_{\mathcal{R}} \psi(v-\mathcal{I} v)\right| & \leq \operatorname{osc}_{1}(\psi)\|\nabla v\| \text { where } \\
\operatorname{osc}_{1}^{2}(\psi) & =\frac{3}{\pi^{2}} \sum_{z \in \mathcal{V}} D_{z}^{2}\left\|\left(\psi-\psi_{z}\right) \ell_{z}^{1 / 2}\right\|_{\omega_{z}}^{2}+3 \sum_{z \in \overline{\mathcal{V}} \backslash \mathcal{V}} \kappa_{z}^{2}\left\|\psi \ell_{z}\right\|_{\omega_{z}}^{2} .
\end{aligned}
$$

Proof. This follows directly from using the continuous Cauchy-Schwarz inequality, followed by the results of Lemma 5.2 with $f=v$, and then the discrete CauchySchwarz inequality. The "extra" factor of 3 in $\operatorname{osc}_{1}(\psi)$ is due to the fact that $\sum_{z \in \overline{\mathcal{V}}}\|\nabla v\|_{\omega_{z}}^{2}=3\|\nabla v\|^{2}$.

We now consider the gradient terms in (5.10). To analyze them we will we collect in the following lemma identities from $[12$ which are most useful to that end.

Lemma 5.4. Let $\omega_{e}$ be the support of the quadratic bump basis function $b_{e}$ associated with edge $e \in \mathcal{E}_{z}$ (see Figure 1), and let $k_{z}$ be constant. Let $J_{e}=J_{e}\left(u_{1}\right)$ denote the jump in the normal derivative of $u_{1}$ accross edge e. For $z \in \mathcal{V}$, (5.14)-(5.17) 


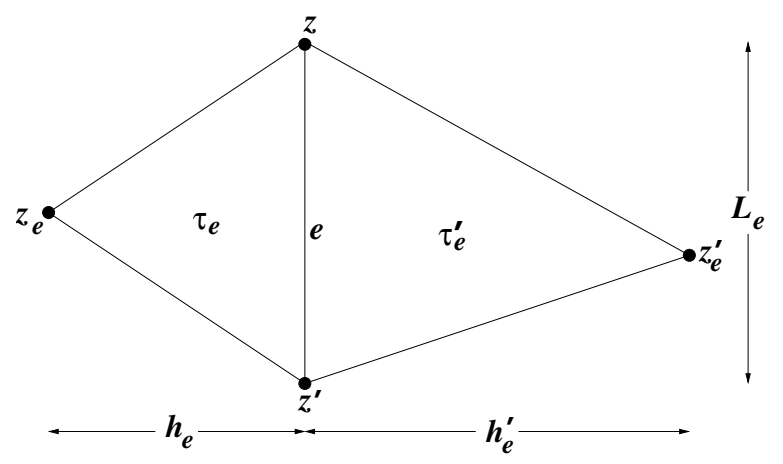

Figure 1. The edge $e \in \mathcal{E}$ and $\omega_{e}=\operatorname{supp}\left(b_{e}\right)$.

hold, and for $z \in \overline{\mathcal{V}} \backslash \mathcal{V}$, (5.14) (5.15) hold:

$$
\begin{aligned}
L_{e} J_{e} & =\frac{3}{2} \int_{\omega_{e}} \nabla u_{1} \cdot \nabla b_{e}=\frac{1}{2} k_{z}\left|\omega_{e}\right|+\frac{3}{2} \int_{\omega_{e}}\left(\psi-k_{z}\right) b_{e}-\nabla \varepsilon \cdot \nabla b_{e} \\
\sum_{e \in \mathcal{E}_{z}} L_{e} J_{e} & =k_{z}\left|\omega_{z}\right|+\frac{3}{2} \sum_{e \in \mathcal{E}_{z}} \int_{\omega_{e}}\left(\psi-k_{z}\right) b_{e}-\nabla \varepsilon \cdot \nabla b_{e} \\
\sum_{e \in \mathcal{E}_{z}} L_{e} J_{e} & =2 \int_{\omega_{z}} \nabla u_{1} \cdot \nabla \ell_{z}=\frac{2}{3} k_{z}\left|\omega_{z}\right|+2 \int_{\omega_{z}}\left(\psi-k_{z}\right) \ell_{z} \\
k_{z}\left|\omega_{z}\right| & =6 \int_{\omega_{z}}\left(\psi-k_{z}\right) \ell_{z}+\frac{9}{2} \sum_{e \in \mathcal{E}_{z}} \int_{\omega_{e}} \nabla \varepsilon \cdot \nabla b_{e}-\left(\psi-k_{z}\right) b_{e} .
\end{aligned}
$$

Proof. These identities are what appear in 12, apart from our choice of sign for $J_{e}$, our notation, and the fact that we have replaced $u_{2}-u_{1}$ in each of the corresponding identities from [12 with $\varepsilon$, which follows from the definition of $\varepsilon$. Identity (5.17) follows from combining (5.15) and (5.16) in such a way as to eliminate the jump terms.

We note that for $z \in \overline{\mathcal{V}}$,

$$
\int_{\omega_{z}} \nabla u_{1} \cdot \nabla\left[\left(v-v_{z}\right) \ell_{z}\right]=\sum_{e \in \mathcal{E}_{z}}\left(\frac{3}{2 L_{e}} \int_{e}\left(v-v_{z}\right) \ell_{z}\right)\left(\int_{\omega_{e}} \nabla u_{1} \cdot \nabla b_{e}\right),
$$

and for $z \in \overline{\mathcal{V}} \backslash \mathcal{V}$

$$
\int_{\omega_{z}} \nabla u_{1} \cdot \nabla\left(v \ell_{z}\right)=\sum_{e \in \mathcal{E}_{z}}\left(\frac{3}{2 L_{e}} \int_{e} v \ell_{z}\right)\left(\int_{\omega_{e}} \nabla u_{1} \cdot \nabla b_{e}\right) .
$$

These identities follow from integration by parts and the first equality in (5.14). We will later use Lemma 5.4 to bound the integrals over $\omega_{e}$, but now we consider the contribution of the edge integrals. 
Lemma 5.5. Let $\tau_{e}$ be a triangle having $e \in \mathcal{E}$ as an edge, $z$ as a vertex, and $z_{e}$ as the vertex opposite e. Let $v \in H^{1}(\mathcal{R})$. We have

$$
\begin{gathered}
\frac{3}{2 L_{e}} \int_{e}\left(v-v_{z}\right) \ell_{z} \leq \sqrt{\frac{27}{16\left|\tau_{e}\right|}}\left\|\left(v-v_{z}\right) \ell_{z}^{1 / 2}\right\|_{\tau_{e}}+\frac{3 g\left(z_{e}, \tau_{e}\right)}{4}\|\nabla v\|_{\tau_{e}}, \\
\frac{3}{2 L_{e}} \int_{e} v \ell_{z} \leq \sqrt{\frac{27}{32\left|\tau_{e}\right|}}\|v\|_{\tau_{e}}+\frac{3 g\left(z_{e}, \tau_{e}\right)}{4}\|\nabla v\|_{\tau_{e}} .
\end{gathered}
$$

Proof. Using (5.4)-(5.6) and the continuous Cauchy-Scwarz inequality, we deduce

$$
\begin{aligned}
\frac{3}{2 L_{e}} \int_{e} f \ell_{z} & =\frac{3}{2 L_{e}} \frac{L_{e}}{2\left|\tau_{e}\right|} \int_{\tau_{e}} 2 f \ell_{z}+\left(x-x_{e}\right) \cdot \nabla\left(f \ell_{z}\right) \\
& =\frac{3}{4\left|\tau_{e}\right|} \int_{\tau_{e}} 3 f \ell_{z}+\left(x-x_{e}\right) \cdot \nabla f \\
& \leq \frac{9}{4\left|\tau_{e}\right|}\left|\int_{\tau_{e}} f \ell_{z}\right|+\frac{3 g\left(z_{e}, \tau_{e}\right)}{4}\|\nabla v\|_{\tau_{e}} .
\end{aligned}
$$

Above, $x_{e}$ are the coordinates of $z_{e}$, which is the vertex opposite $e$ in $\tau_{e}$. The first assertion of the lemma is finally established by choosing $f=v-v_{z}$ and taking the Cauchy-Schwarz bound $\left\|\ell_{z}^{1 / 2}\right\|_{\tau_{e}}\left\|f \ell_{z}^{1 / 2}\right\|_{\tau_{e}}$. The second asserstion is established by choosing $f=v$ and the bound $\left\|\ell_{z}\right\|_{\tau_{e}}\|f\|_{\tau_{e}}$.

Turning now to the gradient integrals over $\omega_{e}$, we see that inserting (5.17) into (5.14) and regrouping terms yields, for $e \in \mathcal{E}$ and $z \in \mathcal{V}$ an endpoint of $e$,

$$
\begin{aligned}
\int_{\omega_{e}} \nabla u_{1} \cdot \nabla b_{e}=\frac{2\left|\omega_{e}\right|}{\left|\omega_{z}\right|} \int_{\omega_{z}}\left(\psi-k_{z}\right) \ell_{z} & +\left(\frac{3\left|\omega_{e}\right|}{2\left|\omega_{z}\right|}-1\right) \int_{\omega_{e}} \nabla \varepsilon \cdot \nabla b_{e}-\left(\psi-k_{z}\right) b_{e} \\
& +\frac{3\left|\omega_{e}\right|}{2\left|\omega_{z}\right|} \sum_{\hat{e} \in \mathcal{E}_{z} \backslash\{e\}} \int_{\omega_{\hat{e}}} \nabla \varepsilon \cdot \nabla b_{\hat{e}}-\left(\psi-k_{z}\right) b_{\hat{e}} .
\end{aligned}
$$

We make the choice $k_{z}=\psi_{z}$, which eliminates the first term in the previous identity. We have implicitly assumed above that all interior edges will have at least one interior vertex as an endpoint. We make this natural and easy-to-enforce assumption throughout. Using the continuous and discrete Cauchy-Schwarz inequalities on (5.20) and (5.14), we obtain

Lemma 5.6. For $e \in \mathcal{E}$ and $z \in \mathcal{V}$ an endpoint of $e$, we have

$$
\begin{aligned}
\left|\int_{\omega_{e}} \nabla u_{1} \cdot \nabla b_{e}\right| & \leq c_{1}(z, e)\|\nabla \varepsilon\|_{\omega_{z}}+c_{2}(\psi, z, e) \text { where } \\
c_{1}^{2}(z, e) & =2\left(\frac{3\left|\omega_{e}\right|}{2\left|\omega_{z}\right|}-1\right)^{2}\left\|\nabla b_{e}\right\|_{\omega_{e}}^{2}+2\left(\frac{3\left|\omega_{e}\right|}{2\left|\omega_{z}\right|}\right)^{2} \sum_{\hat{e} \in \mathcal{E}_{z} \backslash\{e\}}\left\|\nabla b_{\hat{e}}\right\|_{\omega_{\hat{e}}}^{2}, \\
c_{2}(\psi, z, e) & =\left|\frac{3\left|\omega_{e}\right|}{2\left|\omega_{z}\right|}-1\right|\left|\int_{\omega_{e}}\left(\psi-\psi_{z}\right) b_{e}\right|+\frac{4\left|\omega_{e}\right|}{2\left|\omega_{z}\right|} \sum_{\hat{e} \in \mathcal{E}_{z} \backslash\{e\}}\left|\int_{\omega_{\hat{e}}}\left(\psi-\psi_{z}\right) b_{\hat{e}}\right|,
\end{aligned}
$$

and for $z \in \overline{\mathcal{V}} \backslash \mathcal{V}$ and $e \in \mathcal{E}_{z}$, we have

$$
\left|\int_{\omega_{e}} \nabla u_{1} \cdot \nabla b_{e}\right| \leq\left\|\nabla b_{e}\right\|_{\omega_{e}}\|\nabla \varepsilon\|_{\omega_{e}}+\sqrt{\frac{8\left|\omega_{e}\right|}{45}}\|\psi\|_{\omega_{e}} .
$$


The last of these assertions follows directly from the definition of $\varepsilon$ given at the beginning of Section 3 .

We make two remarks before moving on to our main theorem concerning the gradient term (5.10). The first is to make a connection between the integral terms in $c_{2}(\psi, z, e)$ and terms of the sort $\left\|\left(\psi-\psi_{z}\right) \ell_{z}\right\|_{\omega_{z}}$ found in Theorem 5.3. Namely, for $f=\psi-\psi_{z}$, it holds that

$$
\int_{\omega_{e}} f b_{e}=4 \int_{\omega_{e}} f \ell_{z} \ell_{z^{\prime}} \leq 4\left\|\ell_{z}^{1 / 2} \ell_{z^{\prime}}\right\|_{\omega_{e}}\left\|f \ell_{z}^{1 / 2}\right\|_{\omega_{e}}=4 \sqrt{\frac{\left|\omega_{e}\right|}{30}}\left\|f \ell_{z}^{1 / 2}\right\|_{\omega_{e}} .
$$

Here, $z^{\prime}$ is the other endpoint of $e$. Our second remark concerns our treatment of boundary terms $\left\|\psi \ell_{z}\right\|_{\omega_{z}}$ and $\|\psi\|_{\omega_{e}}$ for $z \in \overline{\mathcal{V}} \backslash \mathcal{V}$ and $e \in \mathcal{E}_{z}$ in Theorem 5.3 and Lemma [5.6, and it deserves special notice.

Remark 5.7. Because we are particularly interested in eigenvalue problems in this paper, we have $\psi \in H_{0}^{1}(\mathcal{R})$ and can therefore use (5.12) to obtain bounds on the boundary terms $\left\|\psi \ell_{z}\right\|_{\omega_{z}}$ and $\|\psi\|_{\omega_{e}}$ of the form $\kappa_{z}\|\nabla \psi\|_{S}$ for $S=\omega_{z}$ or $\omega_{e}$. However, for more general $\psi \in H^{1}$, we can still obtain similar bounds for these boundary terms. For example, the first part of Lemma 5.6 can be used for $z \in \overline{\mathcal{V}} \backslash \mathcal{V}$ and $e \in \mathcal{E}_{z}$ by taking the bound $c_{1}\left(z^{\prime}, e\right)\|\nabla \varepsilon\|_{\omega_{z^{\prime}}}+c_{2}\left(\psi, z^{\prime}, e\right)$, where $z^{\prime} \in \mathcal{V}$ is the other endpoint of $e$.

Using Lemmas 5.5] and 5.6, and the discrete Cauchy-Schwarz inequality gives us a theorem concerning the gradient term (5.10).

Theorem 5.8. For $v \in H_{0}^{1}(\mathcal{R})$, there exists a scale invariant constant $C_{1}$, depending only on the mesh $\mathcal{T}_{d}$, such that

$$
\int_{\mathcal{R}} \nabla u_{1} \cdot \nabla(v-\mathcal{I} v) \leq C_{1}\|\nabla \varepsilon\|\|\nabla v\|+\operatorname{osc}_{2}(\psi)\|\nabla v\|
$$

where $\operatorname{osc}_{2}(\psi)$ is defined in the proof below.

Proof. It holds that, for $z \in \mathcal{V}$,

$$
\begin{aligned}
\int_{\omega_{z}} \nabla u_{1} \cdot \nabla\left[\left(v-v_{z}\right) \ell_{z}\right] & \leq \underbrace{\sqrt{\sum_{e \in \mathcal{E}_{z}} c_{1}^{2}(z, e)\left(\frac{27 D_{z}^{2}}{16 \pi^{2}\left|\tau_{e}\right|}+\frac{9 g^{2}\left(z_{e}, \tau_{e}\right)}{16}\right)}}_{c_{1}(z)}\|\nabla \varepsilon\|_{\omega_{z}}\|\nabla v\|_{\omega_{z}} \\
& +\underbrace{\sqrt{\sum_{e \in \mathcal{E}_{z}} c_{2}^{2}(\psi, z, e)\left(\frac{27 D_{z}^{2}}{16 \pi^{2}\left|\tau_{e}\right|}+\frac{9 g^{2}\left(z_{e}, \tau_{e}\right)}{16}\right)}\|\nabla v\|_{\omega_{z}},}_{c_{2}(\psi, z)}
\end{aligned}
$$


and for $z \in \overline{\mathcal{V}} \backslash \mathcal{V}$

$$
\begin{aligned}
\int_{\omega_{z}} \nabla u_{1} \cdot \nabla\left(v \ell_{z}\right) & \leq \underbrace{\max _{e \in \mathcal{E}_{z}} \sqrt{2\left\|\nabla b_{e}\right\|_{\omega_{e}}^{2}\left(\frac{27 \kappa_{z}^{2}}{32\left|\tau_{e}\right|}+\frac{9 g^{2}\left(z_{e}, \tau_{e}\right)}{16}\right)}}_{\tilde{c}_{1}(z)} \sqrt{\sum_{e \in \mathcal{E}_{z}} \frac{\|\nabla \varepsilon\|_{\omega_{e}}^{2}}{2}\|\nabla v\|_{\omega_{z}}} \\
& +\underbrace{\sqrt{\sum_{e \in \mathcal{E}_{z}} \frac{8\left|\omega_{e}\right|}{45}\|\psi\|_{\omega_{e}}^{2}\left(\frac{27 \kappa_{z}^{2}}{32\left|\tau_{e}\right|}+\frac{9 g^{2}\left(z_{e}, \tau_{e}\right)}{16}\right)}}_{\tilde{c}_{2}(\psi, z)}\|\nabla v\|_{\omega_{z}} .
\end{aligned}
$$

Therefore, we have

$$
\begin{aligned}
\int_{\mathcal{R}} \nabla u_{1} \cdot \nabla(v-\mathcal{I} v) & \leq \underbrace{3 \max \left\{\max _{z \in \mathcal{V}} c_{1}(z), \max _{z \in \mathcal{\mathcal { V }} \backslash \mathcal{V}} \tilde{c}_{1}(z)\right\}}_{C_{1}}\|\nabla \varepsilon\|\|\nabla v\| \\
& +\underbrace{\sqrt{3 \sum_{z \in \mathcal{V}} c_{2}^{2}(\psi, z)+3 \sum_{z \in \overline{\mathcal{V}} \backslash \mathcal{V}} \tilde{c}_{2}^{2}(\psi, z)}\|\nabla v\|,}_{\operatorname{osc}_{2}(\psi)}
\end{aligned}
$$

which completes the proof.

Combining Theorems 5.3 and 5.8 we obtain the overall main theorem of this section, and its immediate corollaries.

Theorem 5.9. For $v \in H_{0}^{1}(\mathcal{R})$,

$$
\int_{\mathcal{R}} \nabla\left(u-u_{1}\right) \cdot \nabla v \leq\left(C_{1}\|\nabla \varepsilon\|+\operatorname{osc}(\psi)\right)\|\nabla v\|,
$$

where $\operatorname{osc}(\psi)=\operatorname{osc}_{1}(\psi)+\operatorname{osc}_{2}(\psi)$.

Corollary 5.10. It holds that $\|\nabla \varepsilon\| \leq\left\|\nabla\left(u-u_{1}\right)\right\| \leq C_{1}\|\nabla \varepsilon\|+\operatorname{osc}(\psi)$.

Corollary 5.11. For $\psi \in H_{0}^{1}(\mathcal{R})$, there exists a scale invariant constant $C_{2}$, depending only on the mesh $\mathcal{T}_{d}$, such that $\operatorname{osc}(\psi) \leq C_{2}\|\nabla \psi\| d^{2}$.

This last corollary follows from the definition of $\operatorname{osc}(\psi)$, and the use of (5.11) and Lemma 5.2 with $f=\psi$. For $\psi \in H^{1}(\mathcal{R})$, if we still wanted a bound on the oscillation of the sort above, we would need to handle the boundary terms $z \in \overline{\mathcal{V}} \backslash \mathcal{V}$ slightly differently, in line with Remark 5.7.

The nearest we have found in the literature to bounds of the sort given in Corollary [5.10 is the discussion of Ern and Guermond given on pages 444-445 of [15], but the constants there are not given explicitly. We end this subsection with a few remarks on the above discussion. The first is that Corollary 5.10 replaces both the saturation assumption and the strengthened Cauchy-Schwarz inequality from the traditional analysis of hierarchical basis estimators. For the sake of clarity, we briefly state what the traditional analysis yields for our example. Suppose that there are constants $0<\beta_{1}, \beta_{2}<1$ such that

$$
\begin{aligned}
& \left\|\nabla\left(u-u_{2}\right)\right\| \leq \beta_{1}\left\|\nabla\left(u-u_{1}\right)\right\|, \\
& \int_{\mathcal{R}} \nabla v \cdot \nabla w \leq \beta_{2}\|\nabla v\|\|\nabla w\| \text { for } v \in \mathfrak{L}\left(\mathcal{T}_{d}\right), w \in \mathfrak{B}\left(\mathcal{T}_{d}\right),
\end{aligned}
$$


then we have the bounds

$$
\|\nabla \varepsilon\| \leq\left\|\nabla\left(u-u_{1}\right)\right\| \leq \frac{1}{\sqrt{\left(1-\beta_{1}^{2}\right)\left(1-\beta_{2}^{2}\right)}}\|\nabla \varepsilon\| .
$$

The assumption (5.21) is referred to as the saturation assumption, and its removal from the analysis of error estimators was the motivation of [12] as well as other work. Even though this assumption often holds asymptotically in practice, with $\beta_{1} \rightarrow 0$ as $d \rightarrow 0$, the rate of convergence and the constants involved depend on $u$, and are not readily accessible. At any rate, one cannot disentangle this dependence upon $u$ from $\|\nabla \varepsilon\|$ in (5.23). Maitre and Musy [25] have estimated $\beta_{2}$ solely in terms of the triangulation. In fact, they show that

$$
\beta_{2}^{2} \leq \frac{1}{2}+\frac{1}{3} \max _{\tau \in \mathcal{T}_{d}} \sqrt{\cos ^{2} \theta_{1}+\cos ^{2} \theta_{2}+\cos ^{2} \theta_{3}-\frac{3}{4}} .
$$

In contrast, we have completely eliminated dependencies on any unknown quantity from our constant $C_{1}$. In fact, we even removed dependence upon the known quantity $\varepsilon$ in order to obtain a constant which depends solely on the mesh, by taking the (probably) pessimistic bound

$$
\int_{\omega_{e}} \nabla \varepsilon \cdot \nabla b_{e} \leq\|\nabla \varepsilon\|_{\omega_{e}}\left\|\nabla b_{e}\right\|_{\omega_{e}}
$$

in Lemma [5.6, for every edge in the mesh.

Remark 5.12. Our second remark is that the analysis of nearly all a posteriori error estimates are derived from the fundamental identity (5.7) - notable exceptions being those of gradient recovery type - so the analysis provided here has a good chance of improving many known results, certainly in the sense of making all involved constants explicitly computable. A topic of future work of the second author is to extend the analysis given here to more general elliptic operators and boundary conditions, and other types of error estimates, including some of gradient recovery type.

5.2. The conditioning of the bump stiffness matrix. Finally, we consider the cost of computing the error estimator $\varepsilon\left(\psi, \mathcal{T}_{d}\right) \approx u(\psi)-u_{1}\left(\psi, \mathcal{T}_{d}\right)$. The system matrix for the computation is given by $B_{i j}=\left(\nabla b_{j}, \nabla b_{i}\right)$, where we recall that $b_{k} \in \mathfrak{B}\left(\mathcal{T}_{d}\right)$ is the basis function associated with the interior edge $e_{k}$. The matrix $B$ is certainly larger than the original stiffness matrix $A$ used for computing $u_{1}\left(\psi, \mathcal{T}_{d}\right)$; it can easily have three or four times the number of rows and columns, but never more than five nonzeros per row. However, in contrast to the behavior of $A$, the condition number of $B$ does not deteriorate as the mesh parameter $d$ decreases, and the diagonal of $B$ is such an effective preconditioner that many opt to solve the diagonal system instead. The resulting error estimator $\hat{\varepsilon}\left(\psi, \mathcal{T}_{d}\right)$ does not lose much of its quality (see, for example [2, Ch. 5]), and many consider the compromise for this further speed-up to be worth it. In fact, this is precisely what is done in [29]. In either case, the cost of computing these sorts of error estimates is comparable to other commonly used methods.

The remainder of this subsection is devoted to a more detailed look at the eigenvalues of $B$ and its diagonal $D=\operatorname{diag}(B)$. In particular, we show that they are both spectrally equivalent to the identity (and hence to each other), with reasonable constants of equivalence under reasonable assumptions on the angles in the 
mesh. We first consider the element stiffness matrices $B_{\tau}$, which in our case are given explicitly as

$$
B_{\tau}=\frac{4}{3}\left(\begin{array}{ccc}
\rho & -\cot \theta_{3} & -\cot \theta_{2} \\
-\cot \theta_{3} & \rho & -\cot \theta_{1} \\
-\cot \theta_{2} & -\cot \theta_{1} & \rho
\end{array}\right), \rho=\cot \theta_{1}+\cot \theta_{2}+\cot \theta_{3},
$$

as well as its diagonal $D_{\tau}$. Here and below, $\theta_{i}, i=1,2,3$, are the angles of the triangle. The eigenvalues of $B_{\tau}$ are

$$
\begin{aligned}
\sigma_{k} & =\frac{4}{3}\left(\rho-2 \sqrt{\frac{\cot ^{2} \theta_{1}+\cot ^{2} \theta_{2}+\cot ^{2} \theta_{3}}{3}} \cos \left(\frac{\theta+2(k-1) \pi}{3}\right)\right), \\
\theta & =\arccos \left(\frac{3 \sqrt{3} \cot \theta_{1} \cot \theta_{2} \cot \theta_{3}}{\left(\cot ^{2} \theta_{1}+\cot ^{2} \theta_{2}+\cot ^{2} \theta_{3}\right)^{3 / 2}}\right), k=1,2,3 .
\end{aligned}
$$

It holds that $0<\sigma_{1} \leq \sigma_{3} \leq \sigma_{2}$, and

$$
\begin{array}{cl}
0.744 I \leq B_{\tau} \leq 4.553 I, 2.309 I \leq D_{\tau} \leq 2.667 I & \text { if } \pi / 4 \leq \theta_{k} \leq \pi / 2 \\
0.487 I \leq B_{\tau} \leq 10.373 I, 2.309 I \leq D_{\tau} \leq 5.105 I & \text { if } \pi / 8 \leq \theta_{k} \leq 3 \pi / 4
\end{array} .
$$

Combining these elementwise estimates into estimates for the full matrices $B$ and $D$, we obtain

$$
\begin{aligned}
& 1.488 I \leq B \leq 9.106 I, 4.618 I \leq D \leq 5.334 I \\
& \text { if all angles are between } \pi / 4 \text { and } \pi / 2, \\
& 0.974 I \leq B \leq 20.746 I, 4.618 I \leq D \leq 10.210 I
\end{aligned}
$$

if all angles are between $\pi / 8$ and $3 \pi / 4$.

To obtain the bounds on $B$ from those on $B_{\tau}$ we use that, for $w=\sum_{e \in \mathcal{E}} c_{e} b_{e}$,

$$
\mathbf{c}^{T} B \mathbf{c}=\int_{\mathcal{R}} \nabla w \cdot \nabla w=\sum_{\tau \in \mathcal{T}} \int_{\tau} \nabla w \cdot \nabla w=\sum_{\tau \in \mathcal{T}} \mathbf{c}_{\tau}{ }^{T} B_{\tau} \mathbf{c}_{\tau}
$$

where $\mathbf{c}$ is the vector of coefficients of $w$ with respect to the basis and $\mathbf{c}_{\tau}$ contains only those coefficients corresponding to the (interior) edges of $\tau$. Therefore,

$$
\begin{array}{r}
\lambda_{\min }(B)\|\mathbf{c}\|^{2} \geq \min _{\tau \in \mathcal{T}} \lambda_{\min }\left(B_{\tau}\right) \sum_{\tau \in \mathcal{T}}\left\|\mathbf{c}_{\tau}\right\|^{2}=2 \min _{\tau \in \mathcal{T}} \lambda_{\min }\left(B_{\tau}\right)\|\mathbf{c}\|^{2}, \\
\lambda_{\max }(B)\|\mathbf{c}\|^{2} \leq \max _{\tau \in \mathcal{T}} \lambda_{\max }\left(B_{\tau}\right) \sum_{\tau \in \mathcal{T}}\left\|\mathbf{c}_{\tau}\right\|^{2}=2 \max _{\tau \in \mathcal{T}} \lambda_{\max }\left(B_{\tau}\right)\|\mathbf{c}\|^{2} .
\end{array}
$$

The argument for the diagonal matrices is similar, but simpler. These estimates give a rough sense of what can be expected of the bump stiffness matrix $B$. In particular, we see that $B$ is already pretty well conditioned, and that $D$ provides a very good preconditioner. 

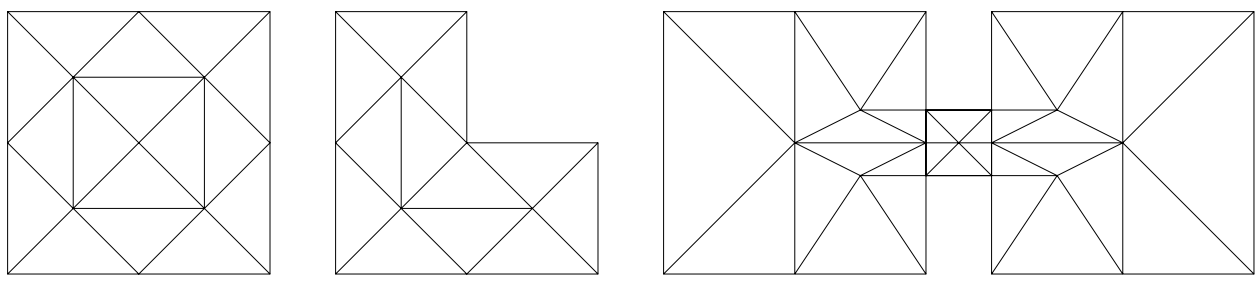

Figure 2. The unit square, L-shaped, and dumbbell domains together with their initial triangulations.

\section{EXPERIMENTS}

In this section we provide experiments for the model problem on three different domains which illustrate the effectivity of our estimates $\eta_{i}\left(\mathfrak{B}_{d}, P_{d}\right)$ of the approximation defects $\eta_{i}\left(P_{d}\right)$, which, in turn, are estimates of the relative eigenvalue and eigenvector errors. In particular, we focus on the quality of our trace-type estimates

$$
E F F=\frac{\sum_{i=1}^{m} \frac{\left|\mu_{i}^{d}-\lambda_{q}\right|}{\mu_{i}^{d}}}{\sum_{i=1}^{m} \eta_{i}^{2}\left(\mathfrak{B}_{d}, P_{d}\right)}
$$

for both single (possibly) degenerate eigenvalues, such as $\lambda_{q}$ in the above equation, or clusters of eigenvalues which may include degenerate members. We recall that our model problem is the Dirichlet Laplace eigenvalue problem: $-\Delta u=\lambda u$ in $\Omega, u=0$ on $\partial \Omega$. The three domains under consideration are the unit square, the L-shaped domain consisting of a concatenation of three unit squares, and the dumbbell domain consisting of two $\pi \times \pi$ squares connected by a $\pi / 4 \times \pi / 4$ square (see Figure 21). The unit square was chosen because of the exact knowledge of its eigenvalues and vectors and the fact that it has many degenerate eigenvalues in the lower part of its spectrum. The L-shaped domain appears frequently in the literature as one of the simplest domains for which analytic solutions of the eigenvalue problem are not generally known. The dumbbell domain provides examples of many pairs (and larger collections) of eigenvalues which just barely miss being degenerate, because the small bridge between the two larger squares has a symmetry-breaking effect. Although exact eigenvalues are not generally known for the latter two domains, Trefethen and Betcke [33. have computed several of them to a high degree of accuracy, and we will use their computed values as the "exact" values in our effectivity tests.

The code used for the experiments was written by the second author in MATLAB, and makes use of its linear solver and eigenvalue solvers - EIGS for the large sparse generalized eigenvalue problems coming from the finite element discretizations, the "backslash" operation 2 for computing the approximate error functions $\varepsilon\left(\mu_{i}^{d} \psi_{i}^{d}, \mathcal{T}_{d}\right)$, and EIG for the small generalized eigenvalue problems needed to compute our estimates of the approximation defects, $\eta_{i}^{2}\left(\mathfrak{B}_{d}, P_{d}\right)$. We also use MATLAB's sparse matrix format. The data structures for the triangulation are trianglebased, and are modeled after those found in PLTMG 4. For adaptive refinement,

\footnotetext{
${ }^{2}$ Because our main emphasis in these experiments is to illustrate the effectivity of our estimator (not necessarily to do things in the fastest possible way), and because the asymptotic behavior of our estimators is observed even for relatively small problems, we have not bothered to use a fast iterative solver for the computation of the $\varepsilon\left(\mu_{i}^{d} \psi_{i}^{d}, \mathcal{T}_{d}\right)$.
} 
we use Rivara's backward-longest-edge bisection algorithm 32 for marked triangles. The marking strategy is based on the local indicators

$$
\sum_{i=1}^{m} \frac{\left\|\nabla \varepsilon\left(\bar{\psi}_{i}^{d}, \mathcal{T}_{d}\right)\right\|_{\tau}^{2}}{\left\|\nabla \varepsilon\left(\bar{\psi}_{i}^{d}, \mathcal{T}_{d}\right)\right\|^{2}+\left\|\nabla u_{1}\left(\bar{\psi}_{i}^{d}, \mathcal{T}_{d}\right)\right\|^{2}},
$$

where $\bar{\psi}_{i}^{d} \in \mathrm{R}\left(P_{d}\right)$ is an argument satisfying

$$
\frac{\left\|\nabla \varepsilon\left(\bar{\psi}_{i}^{d}, \mathcal{T}_{d}\right)\right\|^{2}}{\left\|\nabla \varepsilon\left(\bar{\psi}_{i}^{d}, \mathcal{T}_{d}\right)\right\|^{2}+\left\|\nabla u_{1}\left(\bar{\psi}_{i}^{d}, \mathcal{T}_{d}\right)\right\|^{2}}=\eta_{i}^{2}\left(\mathfrak{B}_{d}, P_{d}\right) .
$$

The triangles whose indicators are larger than the median are marked. Certainly other marking strategies and indicators could be used; if one wished to use a weighting which favored certain approximation defects (relative errors) more heavily than others, then the approach described above could be modified accordingly. Additionally, one could also include local indicators based on the data oscillations (or some suitable simplification of them) for the marking strategy. This might be particularly useful in the early stages of adaptive refinement if larger eigenvalues (with highly oscillatory eigenvectors) are to be approximated.

Remark 6.1. We emphasize that, although the $\eta_{i}^{2}\left(\mathfrak{B}_{d}, P_{d}\right)$ and the corresponding local indicators are computed using the basis for $\mathrm{R}\left(P_{d}\right)$ which is given by the eigensolver, the actual computed quantities are basis-independent. This is a very useful quality to have for error estimation and adaptive refinement, especially in the case of degenerate eigenvalues, because one may have little control over the basis computed by the eigensolver. In particular, the computed bases may "drift" as the mesh is refined either uniformly or adaptively - meaning that, although the bases for two different meshes both span spaces which approximate the true invariant subspace, the bases themselves are only approximately equal up to an orthogonal transformation. Moreover, for solvers such as EIGS, the computed basis may be different for consecutive calls on the same mesh! At any rate, an ideal mesh for a given problem should be well-suited for the entire invariant subspace, and not just to a given basis.

6.1. The unit square. As mentioned above, the eigenvalues and eigenvectors are explicitly known in this case. Namely, we have

$$
\left(k^{2}+n^{2}\right) \pi^{2} \quad \text { paired with } 2 \sin (k \pi x) \sin (n \pi y),
$$

with the eigenfunctions as given above forming an orthonormal basis for the complete eigenspace. For our first experiment, we approximate the smallest six eigenvalues

$$
10 \pi^{2}, 10 \pi^{2}, 8 \pi^{2}, 5 \pi^{2}, 5 \pi^{2}, 2 \pi^{2}
$$

having two simple eigenvalues and two degenerate pairs. In Table 1 we see the computed Ritz values in descending order, together with the effectivity indices (6.1) for our estimates of the relative error in approximating these eigenvalues at various levels of adaptive refinement; $N$ indicates the number of degrees of freedom for the problem. We remark that the asymptotic behavior indicated by (4.4) and (3.13) is observed immediately, with nearly perfect effectivity observed throughout the refinement process. For this experiment and the analogous ones for the other domains, we observe approximately $\mathcal{O}\left(N^{-1}\right)$-convergence, which corresponds to the optimal $\mathcal{O}\left(h^{2}\right)$ convergence rate in the case of quasi-uniform meshes. Although we do not express these computations in table form for this problem, they can be 
deduced from the exact eigenvalues and the information given in Tables 1 , 3 and 4 . and are shown graphically in the case of the L-shaped domain below.

TABLE 1. Eigenvalue estimates and effectivity indices for the unit square.

\begin{tabular}{|c|cccccc|c|}
\hline$N$ & $\mu_{6}$ & $\mu_{5}$ & $\mu_{4}$ & $\mu_{3}$ & $\mu_{2}$ & $\mu_{1}$ & $E F F$ \\
\hline 45 & 113.1352 & 112.4708 & 91.0246 & 53.3258 & 53.3074 & 20.4309 & 1.1270 \\
111 & 106.5602 & 106.1753 & 82.5317 & 50.9809 & 50.9774 & 19.9609 & 1.0852 \\
295 & 101.6302 & 101.3446 & 80.7847 & 50.1042 & 50.0528 & 19.8777 & 1.0742 \\
731 & 99.7023 & 99.6333 & 79.7179 & 49.6299 & 49.6294 & 19.7851 & 1.0634 \\
1765 & 99.2275 & 99.1885 & 79.2009 & 49.4685 & 49.4685 & 19.7592 & 1.0619 \\
4248 & 98.8957 & 98.8811 & 79.1084 & 49.4033 & 49.4031 & 19.7494 & 1.0546 \\
\hline
\end{tabular}

To illustrate the implications of (4.5) and (3.13), we first reconsider the statement of (4.5). The evaluation of the formula (4.5) - for multiple eigenvalues - is not easy within a practical numerical procedure. The problem is that the formula (4.5) assumes that the finite element eigenvalue procedure has returned the Ritz vectors which are matched to the eigenvectors from the invariant subspace in the sense of Proposition 2.5. For practical tests we will study the quotients $\min _{\psi \in \mathrm{R}\left(P_{d}\right)} \| \nabla \psi-$ $\nabla v_{i}\left\|^{2} /\right\| \nabla v_{i} \|^{2}$ rather than the quotients $\left\|\nabla \psi_{i}^{d}-\nabla v_{i}\right\|^{2} /\left\|\nabla v_{i}\right\|^{2}$, which appeared in (4.5). According to the analysis of Beattie [7] these quotients have essentially the same asymptotic behavior. Statement (4.5) can now be expressed as

$$
\lim _{d \rightarrow 0} \frac{\sum_{i=1}^{m} \frac{\min _{\psi \in R\left(P_{d}\right)}\left\|\nabla \psi-\nabla v_{i}\right\|^{2}}{\left\|\nabla v_{i}\right\|^{2}}}{\sum_{i=1}^{m} \eta_{i}^{2}\left(P_{d}\right)}=1,
$$

where we recall that the $v_{i}$ form an orthonormal eigenbasis for the invariant subspace which we are trying to approximate with $\mathrm{R}\left(P_{d}\right)$. A direct computation shows that

$$
\begin{aligned}
\frac{\min _{\psi \in \mathrm{R}\left(P_{d}\right)}\left\|\nabla \psi-\nabla v_{i}\right\|^{2}}{\left\|\nabla v_{i}\right\|^{2}} & =1-\sum_{j=1}^{m} \frac{\lambda_{i}}{\mu_{j}}\left(\int_{\mathcal{R}} v_{i} \psi_{j}^{d}\right)^{2} \\
& =\sin ^{2} \angle\left(v_{i}, \mathrm{R}\left(P_{d}\right)\right)+\sum_{j=1}^{m} \frac{\mu_{j}-\lambda_{j}}{\mu_{j}}\left(\int_{\mathcal{R}} v_{i} \psi_{j}^{d}\right)^{2},
\end{aligned}
$$

so it is clear, at least theoretically, how to compute the numerator in (6.2). In the equation above, $\angle\left(v_{i}, \mathrm{R}\left(P_{d}\right)\right)$ is the angle between $v_{i}$ and the subspace $\mathrm{R}\left(P_{d}\right)$. Furthermore, the result (4.4), together with the $\sin \Theta$ theorem from 20. proves the conclusion (6.2). A second natural measure of the effectivity of our computed $\eta_{i}\left(\mathfrak{B}_{d}, P_{d}\right)$ is

$$
E F F_{2}=\frac{\sum_{i=1}^{m} \frac{\min _{\psi \in \mathrm{R}\left(P_{d}\right)}\left\|\nabla \psi-\nabla v_{i}\right\|^{2}}{\left\|\nabla v_{i}\right\|^{2}}}{\sum_{i=1}^{m} \eta_{i}^{2}\left(\mathcal{B}_{d}, P_{d}\right)},
$$

and we investigate it in the following experiment.

For this experiment, we consider the degenerate eigenpairs

$$
\lambda_{2}=\lambda_{3}=5 \pi^{2}, v_{2}=2 \sin \pi x \sin 2 \pi y, v_{3}=2 \sin 2 \pi x \sin \pi y,
$$


TABLE 2. Eigenvector estimates and effectivity indices for the unit square and the degenerate eigenvalue $\lambda_{2}=\lambda_{3}=5 \pi^{2}$.

\begin{tabular}{|c|ccccccc|}
\hline$N$ & 9 & 29 & 73 & 215 & 589 & 1385 & 3369 \\
$E F F_{2}$ & 1.7255 & 1.1744 & 1.1119 & 1.0721 & 1.0637 & 1.0633 & 1.0534 \\
\hline
\end{tabular}
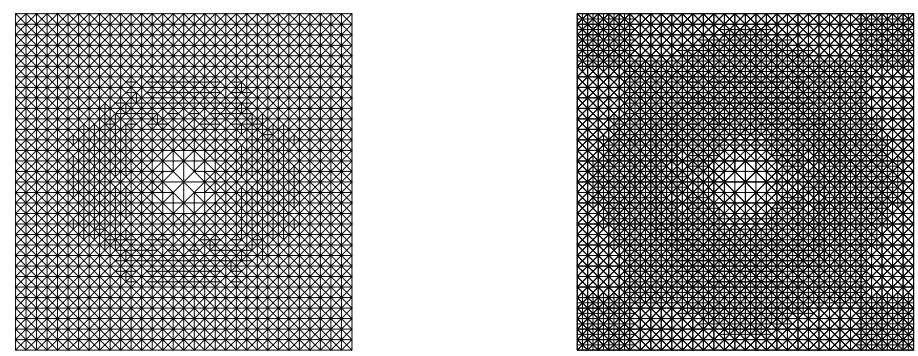

Figure 3. Adaptively refined meshes for the unit square and the degenerate eigenvalue $\lambda_{2}=\lambda_{3}=5 \pi^{2}$, having $N=2193$ and $N=$ 3369 degrees of freedom.

and see how closely (6.4) comes to the predicted asymptotic behavior (6.2). The results of this experiment are given in Table 2. We see that the asymptotically optimal behavior of the $\eta_{i}\left(\mathcal{B}_{d}, P_{d}\right)$ is realized even for coarse meshes. The quantities in the numerator of (6.4) are computed using a twelve-point quadrature rule - which is exact for polynomials of degree six - so the initial effectivity estimates are slightly inflated due to quadrature error. Pictures of the final two adapted meshes for this experiment are given in Figure 3 .

6.2. The L-shaped domain. Although some of the eigenvalues and eigenvectors for the L-shaped domain are known explicitly, most are not. We take the highly accurate values computed by Trefethen and Betcke for the six smallest eigenvalues

$$
\text { 41.474510, 31.912636, 29.521481, } 19.739209\left(2 \pi^{2}\right), 15.197252,9.6397238
$$

and perform the analogous experiment as was done for the square domain. In Table 3, we show the individual $\mathrm{eff}_{k}=r_{k} / \tilde{\eta}_{k}^{2}$ as well as the total effectivity $E F F$. This differs from the way we present tabular data for the Square and Dumbbell problems in Table 1 and Table 4, and we do it to give more explicit information about how well the individual approximation defects $\tilde{\eta}_{k}^{2}$ track the behavior of the individual relative errors $r_{k}=\left|\lambda_{k}-\mu_{k}\right| / \mu_{k}$. This information is also given graphically in Figure 4, were we see that the individual approximation defects $\tilde{\eta}_{k}^{2}$ and their sum $\tilde{\eta}^{2}=\sum \tilde{\eta}_{k}^{2}$ do an excellent job of modeling the behavior of the individual relative errors $r_{k}$ and their sum $r=\sum r_{k}$. We also see in Figure 4 that these relative errors are converging at nearly the optimal rate $\mathcal{O}\left(N^{-1}\right)$; the line with slope -1 is given as a reference. In reference to Table 3, we point out that although we expect $E F F \rightarrow 1$ theoretically, nothing in the experiments is computed exactly, so 1.05 (which is very good) is about as good as we see in practice. It is also clear in the table that the adaptive process, which is aimed at reducing the collective relative 
error $r$ (as opposed to reducing each $r_{k}$ individually), means that some $r_{k}$ may be better approximated by their respective $\tilde{\eta}_{k}^{2}$ than others at each stage. In particular, we see here that $\tilde{\eta}_{6}^{2}$ tends to do the best job of approximating $r_{6}$, while $\tilde{\eta}_{1}^{2}$ tends to do the worst job; although 1.2 is still a respectable effectivity.

TABLE 3. Individual and total effectivity indices for the L-shaped domain.

\begin{tabular}{|c|cccccc|c|}
\hline$N$ & eff $_{6}$ & eff $_{5}$ & eff $_{4}$ & eff $_{3}$ & $\mathrm{eff}_{2}$ & eff $_{1}$ & $E F F$ \\
\hline 36 & 1.1413 & 1.1829 & 1.1780 & 1.1390 & 1.2196 & 1.1092 & 1.1617 \\
110 & 1.1166 & 1.0989 & 1.0870 & 1.0843 & 1.0897 & 1.2348 & 1.1107 \\
272 & 1.0358 & 1.1523 & 1.0914 & 1.0635 & 1.1412 & 1.1683 & 1.0970 \\
680 & 1.0581 & 1.0460 & 1.0534 & 1.0568 & 1.1105 & 1.1788 & 1.0700 \\
1689 & 1.0338 & 1.0856 & 1.0663 & 1.0728 & 1.0664 & 1.1957 & 1.0747 \\
3998 & 1.0280 & 1.0747 & 1.0693 & 1.0669 & 1.0816 & 1.1889 & 1.0718 \\
\hline
\end{tabular}

TABLE 4. Eigenvalue estimates and effectivity indices for the dumbbell domain.

\begin{tabular}{|c|cccccc|c|}
\hline$N$ & $\mu_{6}$ & $\mu_{5}$ & $\mu_{4}$ & $\mu_{3}$ & $\mu_{2}$ & $\mu_{1}$ & $E F F$ \\
\hline 85 & 5.6138 & 5.6138 & 5.5491 & 5.5184 & 2.0846 & 2.0809 & 1.1725 \\
243 & 5.2183 & 5.2128 & 5.0333 & 5.0083 & 1.9967 & 1.9929 & 1.1130 \\
650 & 5.0778 & 5.0765 & 4.9095 & 4.8847 & 1.9759 & 1.9718 & 1.1065 \\
1693 & 5.0279 & 5.0278 & 4.8630 & 4.8345 & 1.9673 & 1.9626 & 1.1058 \\
4274 & 5.0093 & 5.0093 & 4.8428 & 4.8143 & 1.9630 & 1.9583 & 1.0944 \\
\hline
\end{tabular}

6.3. The dumbbell domain. Again we take the smallest six highly accurate eigenvalues computed by Trefethen and Betcke,

$$
\text { 4.9968508, 4.9968371, 4.8298953, 4.8007611, 1.9606830, } 1.9557938
$$

and again we perform the analogous experiment. As noted in 33, the effect of the small "bridge" between the two $\pi \times \pi$ squares is to take the smallest three eigenvalues of a single $\pi \times \pi$ square - namely 5, 5 and 2-and split them into nearly degenerate pairs. In Table 4, we again see excellent effectivity even for coarse triangulations. Figure 5 illustrates how our adaptive refinement procedure captures the singular behavior of the invariant subspace associated with the six smallest eigenvalues (particularly eigenvalues 3 and 4 ). 

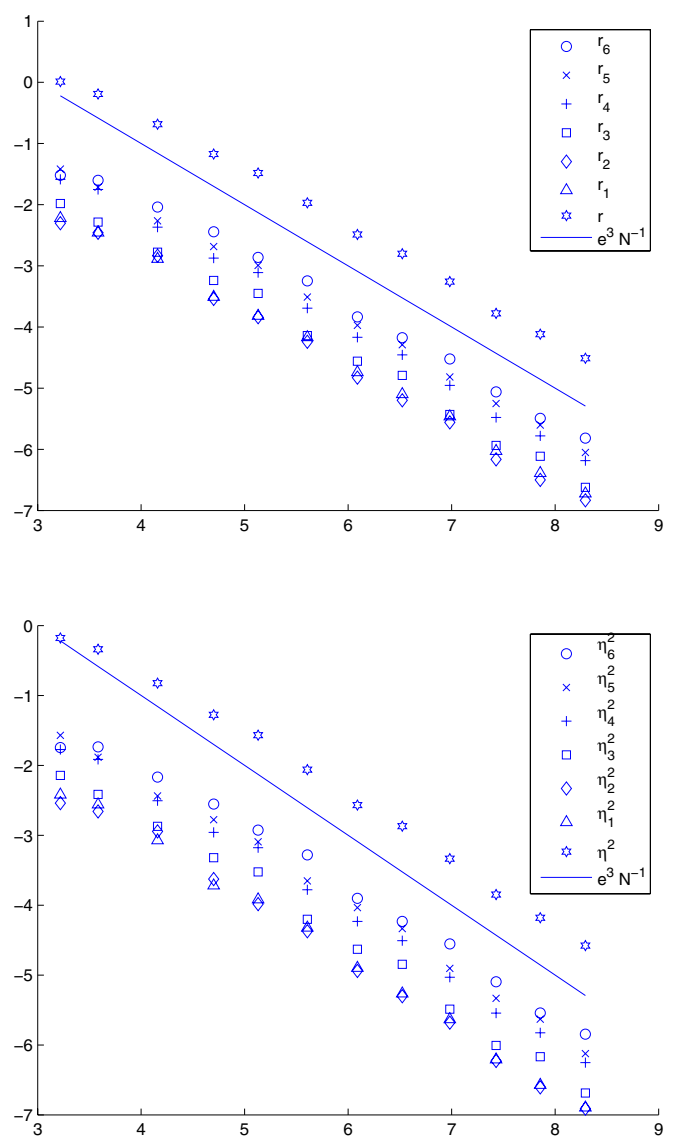

FiguRE 4. Log-log plots showing the convergence of the relative eigenvalue errors (top) as well as the their corresponding approximation defects for the L-shaped domain.

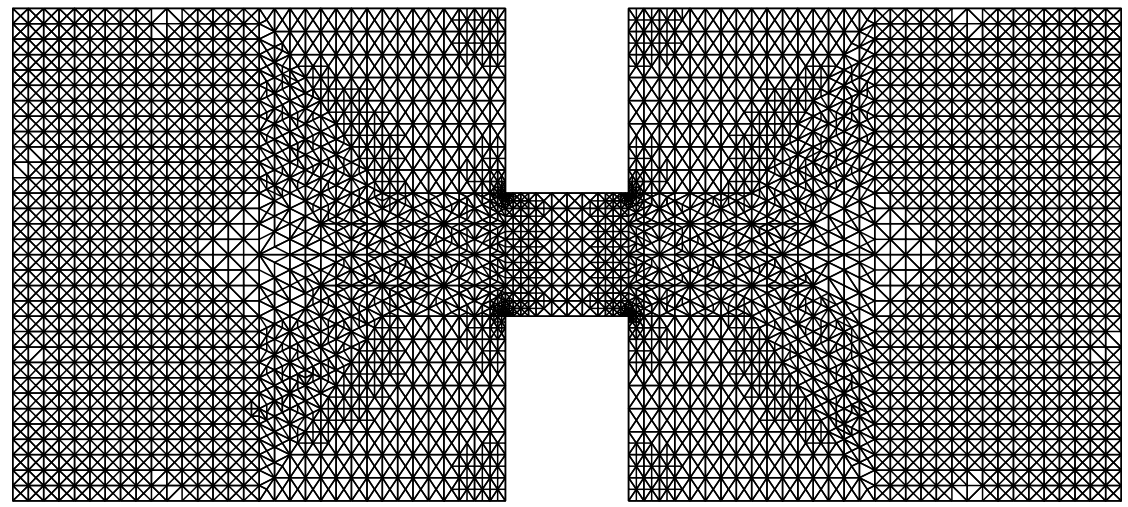

Figure 5. Adaptively refined meshes for the dumbbell domain, having $N=4274$ degrees of freedom. 


\section{CONClusion}

The primary goals of this paper were two-fold:

(1) To establish the equivalence of the approximation defects $\eta_{i}\left(P_{d}\right)$ and the corresponding relative eigenvalue and eigenvector errors.

(2) To provide a practical means of estimating these approximation defects which is provably effective and reliable.

With regard to the first aim, asymptotic exactness was proven in a very general setting, and detailed bounds were also given which always hold. The definition of the approximation defects is such that it is natural to derive estimates for them using the well-developed theory of a posteriori error estimation for elliptic boundary value problems. In principle, one could incorporate a number of different $a$ posteriori techniques in our framework, and we mentioned the use of gradient recovery techniques explicitly, but our focus was on estimates of hierarchical type, $\eta_{i}\left(\mathfrak{B}_{d}, P_{d}\right) \approx \eta_{i}\left(P_{d}\right)$. For this type of estimator we asserted asymptotic exactness for the model problem on convex domains, and also gave detailed bounds which always hold - complementing the results mentioned above. Experiments verified the effectivity of our estimates of the approximation defects as trustworthy indicators of relative eigenvalue/eigenvector errors. In addition to the strengths of our approach mentioned above, we highlight three more. The case of degenerate eigenvalues is treated very naturally in our framework, requiring no special modification, and we need no assumptions concerning the convexity/non-convexity of the domain. Additionally, the approximation defects and their estimates truly are basis-independent, so one truly obtains information about how well the subspace $\mathrm{R}\left(P_{d}\right)$ - given in terms of some basis by whatever eigensolver is used - approximates the true invariant subspace of interest.

We finish with a brief outlook for future work in this area. All of our analysis was done in the context of piecewise linear finite elements, and the analysis of our hierarchical basis estimates was carried out only for the Laplacian with zero Dirichlet conditions. One clear direction in which our results can be extended is to consider more general elliptic operators and boundary conditions. Item (1) above is already dealt with in principle by the arguments given in this paper, so further work in this direction is really to prove something analogous to Theorem 3.1 in the more general setting. Some of the necessary modifications to our arguments are obvious, but others will require a more detailed look. Another area of future work is to provide similar analysis for other eigenvalue approximation methods, such as those arising from $h p$-finite element discretizations. The $h p$-approach is in particular well-suited for eigenvalue problems, because of the higher order of smoothness of the eigenfunctions away from (nonconvex) boundaries and regions of discontinuity of the coefficients of the differential operator.

\section{ACKNOWLEDGEMENTS}

The authors would like to thank Professor Dr. Volker Enss, Aachen and Professor Dr. Wolfgang Hackbusch, Leipzig for helpful discussions and support during the research and the preparation of this manuscript. L. Grubišić was partially supported by the grant: "Spectral decompositions - numerical methods and applications", Grant Nr. 037-0372783-2750 of the Croatian MZOS. Support of the 
Graduate School: "Hierarchy and Symmetry in Mathematical Models", RWTH Aachen University is also kindly acknowledged.

\section{REFERENCES}

[1] G. Acosta and R. G. Durán. An optimal Poincaré inequality in $L^{1}$ for convex domains. Proc. Amer. Math. Soc., 132(1):195-202 (electronic), 2004. MR2021262 (2004j:26031)

[2] M. Ainsworth and J. T. Oden. A posteriori error estimation in finite element analysis. Pure and Applied Mathematics (New York). Wiley-Interscience [John Wiley \& Sons], New York, 2000. MR 1885308 (2003b:65001)

[3] R. E. Bank. Hierarchical bases and the finite element method. In Acta numerica, 1996, volume 5 of Acta Numer., pages 1-43. Cambridge Univ. Press, Cambridge, 1996. MR1624587 (99f:65161)

[4] R. E. Bank. PLTMG: A software package for solving elliptic partial differential equations, users' guide 9.0. Technical report, University of California, San Diego, 2004.

[5] R. E. Bank and J. Xu. Asymptotically exact a posteriori error estimators. I. Grids with superconvergence. SIAM J. Numer. Anal., 41(6):2294-2312 (electronic), 2003. MR2034616 (2004k:65194)

[6] R. E. Bank and J. Xu. Asymptotically exact a posteriori error estimators. II. General unstructured grids. SIAM J. Numer. Anal., 41(6):2313-2332 (electronic), 2003. MR2034617 (2004m:65212)

[7] C. Beattie. Galerkin eigenvector approximations. Math. Comp., 69(232):1409-1434, 2000. MR.1681128 (2001a:65131)

[8] M. Bebendorf. A note on the Poincaré inequality for convex domains. Z. Anal. Anwendungen, 22(4):751-756, 2003. MR2036927 (2004k:26025)

[9] C. Carstensen. Quasi-interpolation and a posteriori error analysis in finite element methods. M2AN Math. Model. Numer. Anal., 33(6):1187-1202, 1999. MR1736895 (2001a:65135)

[10] C. Carstensen and S. A. Funken. Constants in Clément-interpolation error and residual based a posteriori error estimates in finite element methods. East-West J. Numer. Math., 8(3):153175, 2000. MR.1807259 (2002a:65173)

[11] S.-K. Chua and R. L. Wheeden. Estimates of best constants for weighted Poincaré inequalities on convex domains. Proc. London Math. Soc. (3), 93(1):197-226, 2006. MR2235947 (2006m:26030)

[12] W. Dörfler and R. H. Nochetto. Small data oscillation implies the saturation assumption. Numer. Math., 91(1):1-12, 2002. MR1896084(2003e:65195)

[13] Z. Drmač and K. Veselić. New fast and accurate Jacobi SVD algorithm: II. SIAM J. Matrix Anal. Appl., to appear. Preprint LAPACK Working Note 170.

[14] R. G. Durán, C. Padra, and R. Rodríguez. A posteriori error estimates for the finite element approximation of eigenvalue problems. Math. Models Methods Appl. Sci., 13(8):1219-1229, 2003. MR1998821 (2004f:65173)

[15] A. Ern and J.-L. Guermond. Theory and practice of finite elements, volume 159 of Applied Mathematical Sciences. Springer-Verlag, New York, 2004. MR2050138 (2005d:65002)

[16] L. Grubišić. Ritz value estimates and applications in Mathematical Physics. Ph.D. Thesis, Fernuniversität in Hagen, 2005. Available through dissertation.de Verlag im Internet.

[17] L. Grubišić. On eigenvalue estimates for nonnegative operators. SIAM J. Matrix Anal. Appl., 28(4):1097-1125, 2006. MR2276556

[18] L. Grubišić. A posteriori estimates for eigenvalue/vector approximations. PAMM Proc. Appl. Math. Mech., 6(1):59-62, 2006.

[19] L. Grubišić. On Temple-Kato like inequalities and applications. submitted. 2005-Preprint available from http://arxiv.org/abs/math/0511408.

[20] L. Grubišić and K. Veselić. On weakly formulated Sylvester equation and applications. Integral Equations and Operator Theory, 58(2):175-204, 2007. MR2324886

[21] W. Hackbusch. On the computation of approximate eigenvalues and eigenfunctions of elliptic operators by means of a multi-grid method. SIAM J. Numer. Anal., 16(2):201-215, 1979. MR.526484 (80d:65065)

[22] V. Heuveline and R. Rannacher. A posteriori error control for finite approximations of elliptic eigenvalue problems. Adv. Comput. Math., 15(1-4):107-138 (2002), 2001. A posteriori error estimation and adaptive computational methods. MR 1887731 (2002m:65120) 
[23] T. Kato. Perturbation theory for linear operators. Springer-Verlag, Berlin, second edition, 1976. Grundlehren der Mathematischen Wissenschaften, Band 132. MR0407617 (53:11389)

[24] M. G. Larson. A posteriori and a priori error analysis for finite element approximations of self-adjoint elliptic eigenvalue problems. SIAM J. Numer. Anal., 38(2):608-625 (electronic), 2000. MR1770064 (2001e:65174)

[25] J.-F. Maitre and F. Musy. The contraction number of a class of two-level methods; an exact evaluation for some finite element subspaces and model problems. In Multigrid methods (Cologne, 1981), volume 960 of Lecture Notes in Math., pages 535-544. Springer, Berlin, 1982. MR685787 (84g:65153)

[26] D. Mao, L. Shen, and A. Zhou. Adaptive finite element algorithms for eigenvalue problems based on local averaging type a posteriori error estimates. Adv. Comput. Math., 25(1-3):135160, 2006. MR2231699 (2007d:65103)

[27] P. Morin, R. H. Nochetto, and K. G. Siebert. Convergence of adaptive finite element methods. SIAM Rev., 44(4):631-658 (electronic) (2003), 2002. Revised reprint of "Data oscillation and convergence of adaptive FEM" SIAM J. Numer. Anal. 38 (2000), no. 2, 466-488 (electronic); MR.1770058 (2001g:65157)

[28] P. Morin, R. H. Nochetto, and K. G. Siebert. Local problems on stars: a posteriori error estimators, convergence, and performance. Math. Comp., 72(243):1067-1097 (electronic), 2003. MR.1972728 (2004d:65129)

[29] K. Neymeyr. A posteriori error estimation for elliptic eigenproblems. Numer. Linear Algebra Appl., 9(4):263-279, 2002. MR1909253 (2003c:65111)

[30] J. S. Ovall. Function, gradient, and Hessian recovery using quadratic edge-bump functions. SIAM J. Numer. Anal., 45(3):1064-1080 (electronic), 2007. MR2318802 (2008e:65363)

[31] L. E. Payne and H. F. Weinberger. An optimal Poincaré inequality for convex domains. Arch. Rational Mech. Anal., 5:286-292 (1960), 1960. MR0117419(22:8198)

[32] M.-C. Rivara. New longest-edge algorithms for the refinement and/or improvement of unstructured triangulations. Internat. J. Numer. Methods Engrg., 40(18):3313-3324, 1997. MR 1471613

[33] L. N. Trefethen and T. Betcke. Computed eigenmodes of planar regions. In Recent advances in differential equations and mathematical physics, volume 412 of Contemp. Math., pages 297-314. Amer. Math. Soc., Providence, RI, 2006. MR2259116 (2008a:35042)

[34] R. Verfürth. A review of a posteriori error estimation and adaptive mesh refinement techniques. Wiley-Teubner Series Advances in Numerical Mathematics. John Wiley \& Sons Ltd., Chichester, 1996.

[35] R. Verfürth. Error estimates for some quasi-interpolation operators. M2AN Math. Model. Numer. Anal., 33(4):695-713, 1999. MR1726480(2001a:65149)

Institut für Reine und angewandte Mathematik, RWTH-Aachen, Templergraben 52, D-52062 Aachen, Germany

Current address: Department of Mathematics, Univ-Zagreb, Bijenicka 30, 10000 Zagreb, Croatia

E-mail address: luka.grubisic@math.hr

Max-Planck-Institut für Mathematik in Den Naturwissenschaften, Inselstr. 22-26, D-04103 Leipzig, Germany

E-mail address: ovall@mis.mpg.de

Current address: California Institute of Technology, Pasadena, California 91125-5000

E-mail address: jovall@acm.caltech.edu 